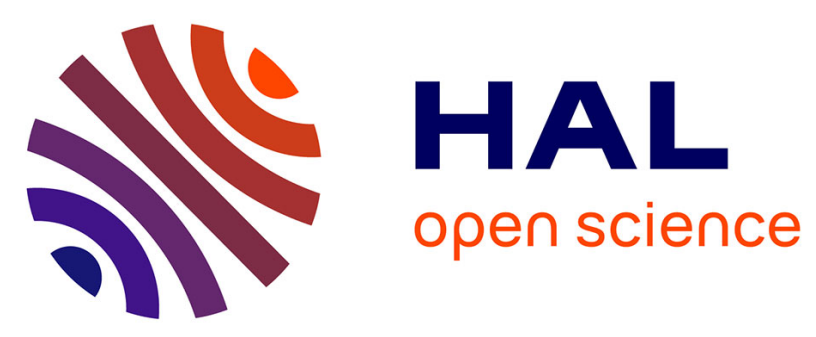

\title{
A modal-based approach to the nonlinear vibration of strings against a unilateral obstacle: Simulations and experiments in the pointwise case
}

Clara Issanchou, Stefan Bilbao, Jean-Loic Le Carrou, Cyril Touzé, Olivier Doaré

\section{To cite this version:}

Clara Issanchou, Stefan Bilbao, Jean-Loic Le Carrou, Cyril Touzé, Olivier Doaré. A modalbased approach to the nonlinear vibration of strings against a unilateral obstacle: Simulations and experiments in the pointwise case. Journal of Sound and Vibration, 2017, 393, pp.229-251. 10.1016/j.jsv.2016.12.025 . hal-01461730

\author{
HAL Id: hal-01461730 \\ https://hal.science/hal-01461730
}

Submitted on 23 Feb 2017

HAL is a multi-disciplinary open access archive for the deposit and dissemination of scientific research documents, whether they are published or not. The documents may come from teaching and research institutions in France or abroad, or from public or private research centers.
L'archive ouverte pluridisciplinaire HAL, est destinée au dépôt et à la diffusion de documents scientifiques de niveau recherche, publiés ou non, émanant des établissements d'enseignement et de recherche français ou étrangers, des laboratoires publics ou privés. 


\title{
A modal-based approach to the nonlinear vibration of strings against a unilateral obstacle: simulations and experiments in the pointwise case.
}

\author{
Clara Issanchou ${ }^{\mathrm{a}, *}$, Stefan Bilbao ${ }^{\mathrm{c}}$, Jean-Loïc Le Carrou ${ }^{\mathrm{a}}$, Cyril Touzé $^{\mathrm{b}}$, Olivier Doaré ${ }^{\mathrm{b}}$ \\ ${ }^{a}$ Sorbonne Universités, UPMC Univ Paris 06, CNRS, LAM / Institut D'Alembert, 4 place Jussieu, 75252 Paris Cedex \\ 05, France \\ ${ }^{b}$ IMSIA, ENSTA Paris Tech-CNRS-EDF-CEA, Université Paris Saclay, 828 Boulevard des Maréchaux, 91762 \\ Palaiseau Cedex \\ ${ }^{c}$ Acoustics and Audio Group. James Clerk Maxwell Building, King's Buildings, University of Edinburgh, Edinburgh, \\ EH9 $3 J Z$
}

\begin{abstract}
This article is concerned with the vibration of a stiff linear string in the presence of a rigid obstacle. A numerical method for unilateral and arbitrary-shaped obstacles is developed, based on a modal approach in order to take into account the frequency dependence of losses in strings. The contact force of the barrier interaction is treated using a penalty approach, while a conservative scheme is derived for time integration, in order to ensure long-term numerical stability. In this way, the linear behaviour of the string when not in contact with the barrier can be controlled via a mode by mode fitting, so that the model is particularly well suited for comparisons with experiments. An experimental configuration is used with a point obstacle either centered or near an extremity of the string. In this latter case, such a pointwise obstruction approximates the end condition found in the tanpura, an Indian stringed instrument. The second polarisation of the string is also analysed and included in the model. Numerical results are compared against experiments, showing good accuracy over a long time scale.
\end{abstract}

Keywords: Numerical methods, 3D string vibration, experimental study, unilateral contact, sound synthesis, tanpura

\section{Introduction}

The problem of vibrating media constrained by a unilateral obstacle is a longstanding problem which has been under study for more than a century [1, 2]. Indeed, the first important developments can be attributed to Hertz with his formulation of a general law for the contact between elastic solids in 1881 [3. Since then, applications of contact mechanics can be found in such diverse fields as e.g. computer graphics [4, for instance for simulating the motion of hair [5]; to human joints in biomechanics [6] or component interactions in turbines [7, 8]. A particular set of applications is found in musical acoustics, where collisions are of prime importance in order to fully understand and analyse the timbre of musical instruments [9, 10, 11. Within this framework, the problem of a vibrating string with a unilateral constraint, as a key feature of numerous instruments, is central and is particularly important to the sound of Indian instruments [12, 13, 14, and also in the string/fret contact in fretted instruments [15, 16].

The first studies on a vibrating string with a unilateral constraint were restricted to the case of an ideal string with a rigid obstacle in order to derive analytical and existence results [17, 18, 19, 20, 21]. In particular, solutions to the cases of a centered point obstacle, a plane obstacle and a few continuous obstacles have been obtained explicitly. Existence and uniqueness of the solution to the non-regularised problem has been shown in the case of a string vibrating against point obstacles [22] and of a concave obstacle if conservation of energy is imposed [20. There are no general results when the obstacle is convex. Moreover, Schatzman proved that the penalised problem with a point obstacle converges to

\footnotetext{
* Corresponding author

Email address: issanchou@lam.jussieu.fr (Clara Issanchou )
} 
the non-regularised problem 22 . The pointwise case is thus well-understood theoretically, and various interesting properties have been demonstrated as mentioned above.

In addition, numerical studies have been undertaken to simulate collisions for more realistic string models by including various effects, such as dispersion. Existing numerical methods include digital waveguides [23, 24, 25], sometimes coupled with finite differences [26] in the case of an ideal string, and for a stiff damped string interacting with an obstacle located at one end of the string [14]. Other models are based on a modal description of the string motion, as in [27] where an ideal string vibrating against a parabolic obstacle at one boundary is considered, under the assumption of perfect wrapping of the string on the bridge as in [28. However, the existence of multiple contacts as a necessary condition for simulating the sound of sitar has been established in [27, 29]. Contacts between a string and point obstacles are modelled with a modal approach in [30 for a dispersive lossy string against a tanpura-like bridge. The functional transformation method (FTM) is used in 15 for a string interacting with frets. In the latter study, damping model is controlled by a few parameters only. Interaction between a continuous system and a point obstacle is also modelled in [29], using a modal coefficient of restitution (CoR) method [31, 32, assuming infinitesimal contact times.

More recently, energy-based methods have been developed, allowing the simulation of stiff lossy strings against an arbitrarily shaped obstacle. Hamilton's equations of motion are discretised in [33, and the case of the tanpura bridge is derived in [34. Finite difference methods are used in [11] and the special case of the interaction between a string and a fretboard is detailed in [16. In these latter models, eigenfrequencies and damping parameters cannot be arbitrary, but follow a distribution tuned through a small number of parameters. In addition, these studies consider only one transverse motion of the string, and numerical dispersion effects appear due to the use of finite difference approximations.

The inclusion of the two transverse polarisations in the modeling of vibrating strings with contact is also seldom seen in the literature. A first attempt has been proposed in 35 for the case of the violin, where finite differences are employed to model a linear bowed string motion, including interactions between the string and fingers as well as the fingerboard. Early developments are also shown in [36, extending the study presented in [33]. However, numerical results are not compared to experimental measurements of the string motion.

Whereas an abundant literature exists on numerical simulations of a string vibrating against an obstacle, only a few experimental studies have been carried out. Research on isolated strings is detailed in [37, 30, and measurements on complete instruments are presented in [38, 39, 14, highlighting the influence of the obstacle shape and position on the timbral richness of sounds. However a detailed comparison of experiments with numerical results in order to understand the relative importance of modeling features such as e.g. dispersion, nonlinearity and damping due to contact has not been carried out.

The aim of this paper is twofold. First, an accurate and flexible numerical method is developed in Section 2. The distinctive feature of the approach is that it relies on a modal description, in order to take into account any frequency dependence of the losses, and also in order to eliminate any effect of numerical dispersion. The contact law is formulated in terms of a penalty potential and an energyconserving scheme is derived, adapted to the modal-based approach. The convergence of the outcomes of the models is then thoroughly studied in Section 3 for a pointwise obstacle, with a comparison to an analytical solution. The second main objective of the study is to compare simulations with experiments. For that purpose, the experimental protocol is presented in Section 4. The versatility of the numerical method is illustrated with a mode by mode fitting of the measured linear characteristics (eigenfrequencies and modal damping factors). Comparisons with experiments are conducted in Section 5 for two different point obstacles, located either at the string centre or near one extremity of the string. The second polarisation is also measured and compared to the outcomes of a simple model incorporating the horizontal vibration in Section 5.2 .3 .

\section{Theoretical model and numerical implementation}

\subsection{Continuous model system}

The vibrating structure considered here is a stiff string of length $L(\mathrm{~m})$, tension $T\left(\mathrm{~N} \cdot \mathrm{m}^{-1}\right)$, and with linear mass density $\mu\left(\mathrm{kg} \cdot \mathrm{m}^{-1}\right)$. The stiffness is described by the Young's modulus $E(\mathrm{~Pa})$ of the material and the moment of inertia associated with a circular cross-section $I=\pi r^{4} / 4$, where $r$ is the string radius $(\mathrm{m})$. The string is assumed to vibrate in the presence of an obstacle described by a 
fixed profile $g(x), x \in[0, L]$, located under the string at rest (see Fig. 1). The obstacle is assumed to be of constant height along $(O y)$. In the remainder of the paper, it is said to be a point obstacle when it is a point along $(O x)$, however it still has a constant height along $(O y)$.

In this section we restrict ourselves to the vertical $(O z)$-polarisation. The second, horizontal polarisation is taken into account in Section 2.7

The transverse displacement $u(x, t)$ of the string along $(O z)$ is governed by the following equation, under the assumption of small displacements:

$$
\mu u_{t t}-T u_{x x}+E I u_{x x x x}=f
$$

where the subscript $t$ (respectively $x$ ) refers to a partial derivative with respect to time (respectively space). The right-hand side term $f(x, t)$ refers to the external contact force per unit length exerted by the barrier on the string. Simply supported boundary conditions are assumed, which are commonly used for musical strings having a weak stiffness [30, 40]:

$$
u(0, t)=u(L, t)=u_{x x}(0, t)=u_{x x}(L, t)=0, \quad \forall t \in \mathbb{R}^{+} .
$$

No damping is included so far; a detailed model of loss will be introduced once modal analysis has been performed, see Section 2.3.1.

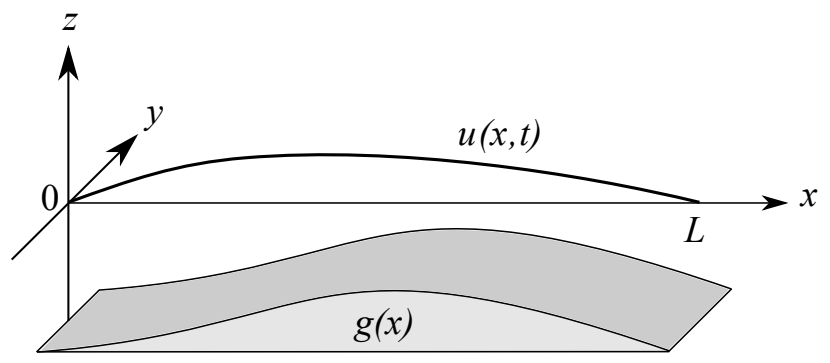

Figure 1: A string of length $L$ vibrating against an obstacle $g(x)$.

The contact force density $f(x, t)$ vanishes as long as the string does not collide with the barrier. The model to be employed here relies on a penalty approach where a small amount of interpenetration is modeled. Penalisation methods are to be viewed in contrast with nonsmooth methods for which no penetration is allowed [41, 42]. In order to derive a general framework, a two-parameter family of power-law expression for the contact force is used:

$$
f(x, t)=f(\eta(x, t))=K[\eta(x, t)]_{+}^{\alpha},
$$

where $\eta(x, t)=g(x)-u(x, t)$ is a measure of interpenetration of the string into the barrier, and $[\eta]_{+}=\frac{1}{2}(\eta+|\eta|)$ is the positive part of $\eta$. This formulation allows the representation of a Hertz-like contact force, where the coefficients $K$ and $\alpha$ can be tuned depending on the material in contact 43, 44, 45]. This interaction model has already been used in the realm of musical acoustics for various interactions-see e.g. [11, 46, 47, 10, 33. In [47] for example, it is used to model the hammer-string interaction in the piano, where the contact model describes the compression of the hammer felt. In the present case of the string colliding with a rigid body, the force expression represents a penalisation of the interpenetration that should remain small; as such, large values of $K$ as compared to the tension and shear restoring forces has to be selected. In the literature, current values used in numerical simulations for this problem are in the range of $10^{7}$ to $10^{15}$, see e.g. [33, 16, 11.

\subsubsection{Energy balance}

The continuous total energy of the system is detailed here. Energy considerations will be useful in the remainder of the study in order to derive an energy-conserving and stable numerical scheme.

The contact force density (3) derives from a potential $\psi$ :

$$
f=\frac{\mathrm{d} \psi}{\mathrm{d} \eta}, \quad \text { with } \quad \psi(\eta)=\frac{K}{\alpha+1}[\eta]_{+}^{\alpha+1} .
$$


The total energy of the system may be obtained by multiplying the equation of motion (1) by the velocity $u_{t}$. Employing integration by parts, one obtains the expression of the continuous energy:

$$
\mathscr{H}=\int_{0}^{L}\left[\frac{\mu}{2}\left(u_{t}\right)^{2}+\frac{T}{2}\left(u_{x}\right)^{2}+\frac{E I}{2}\left(u_{x x}\right)^{2}+\psi\right] \mathrm{d} x .
$$

It satisfies $\mathscr{H} \geq 0$ and the following equality:

$$
\frac{\mathrm{d} \mathscr{H}}{\mathrm{d} t}=0
$$

implying that energy is conserved. The first three terms in (5) correspond respectively to the stored energy due to inertia, tension and stiffness. The final term denotes the energy stored in the contact mechanism under compression.

\subsection{Modal approach}

The eigenproblem related to Eq. (1) without contact force $f$ consists of finding the functions $\phi_{j}(x)$ which are the solutions of:

$$
-T \phi_{j}^{\prime \prime}+E I \phi_{j}^{\prime \prime \prime \prime}-\mu \omega_{j}^{2} \phi_{j}=0,
$$

where' designates the spatial derivative, together with the boundary conditions given in Eq. 2. The normal modes are thus:

$$
\phi_{j}(x)=\sqrt{\frac{2}{L}} \sin \left(\frac{j \pi x}{L}\right) \quad \text { for } \quad j \geq 1,
$$

and are orthogonal and normalised such that $\int_{0}^{L} \phi_{j}(x) \phi_{k}(x) \mathrm{d} x=\delta_{j k}$.

The unknown displacement $u(x, t)$, when truncated to $N_{m}$ modes, may be written as $\hat{u}(x, t)$, defined as

$$
\hat{u}(x, t)=\sum_{j=1}^{N_{m}} q_{j}(t) \phi_{j}(x),
$$

where $q_{j}(t)$ is the $j^{t h}$ modal amplitude. For simplicity, the hat notation is dropped in the remainder of the paper.

Writing $u$ as its expansion in (1) and using the orthogonality, one obtains:

$$
\mu\left(\ddot{\mathbf{q}}+\mathbf{\Omega}^{2} \mathbf{q}\right)=\mathbf{F},
$$

where the vector $\mathbf{q}=\left[\mathbf{q}_{\mathbf{1}}, \ldots, \mathbf{q}_{\mathbf{N}_{\mathbf{m}}}\right]^{\mathbf{T}}$ contains modal coefficients, $\ddot{\mathbf{q}}$ is its second time derivative and $\boldsymbol{\Omega}$ is a diagonal matrix such that $\Omega_{j j}=\omega_{j}=2 \pi \nu_{j}$.

Eigenfrequencies are given by $\nu_{j}=j \frac{c_{0}}{2 L} \sqrt{1+B j^{2}}$, where $c_{0}=\sqrt{\frac{T}{\mu}}$ is the wave velocity and $B=\frac{\pi^{2} E I}{T L^{2}}$ describes the inharmonicity created by taking into account the stiffness of the string. Finally the righthand side vector $\mathbf{F}$ represents the modal projection of the contact force, with $F_{j}=\int_{0}^{L} f(x, t) \phi_{j}(x) \mathrm{d} x$.

\subsection{Losses}

In this section, a standard model of string damping mechanisms is reviewed. Damping due to air friction and internal losses are first presented, then losses due to contact phenomena are modeled.

\subsubsection{Air friction and internal losses}

One advantage of using a modal approach is that damping parameters can be tuned at ease, as recently used in [48] for the nonlinear vibrations of plates with the purpose of synthesising the sound of gongs. A lossless string is described in equation (10), where the linear part corresponds to the description of a lossless oscillator for each mode. Damping can therefore be introduced by generalising each mode to a lossy oscillator. Eq. (10) thus becomes:

$$
\mu\left(\ddot{\mathbf{q}}+\mathbf{\Omega}^{2} \mathbf{q}+2 \Upsilon \dot{\mathbf{q}}\right)=\mathbf{F}
$$

where $\Upsilon$ is a diagonal matrix such that $\Upsilon_{j j}=\sigma_{j} \geq 0$. A damping parameter $\sigma_{j}$ is thus associated to each modal equation. 
In this contribution, the frequency dependence of losses is taken into account using the theoretical model proposed by Valette and Cuesta [30]. This model is especially designed for strings, and shows a strong frequency dependence that cannot be expressed easily in the time domain. It describes the three main effects accounting for dissipation mechanisms in strings, namely friction with the surrounding acoustic field, viscoelasticity and thermoelasticity of the material. The following expression of the quality factor $Q_{j}=\pi \nu_{j} / \sigma_{j}$ is introduced:

$$
Q_{j}^{-1}=Q_{j, \text { air }}^{-1}+Q_{j, \mathrm{ve}}^{-1}+Q_{\mathrm{te}}^{-1},
$$

where subscripts air, ve and te refer, respectively, to frictional, viscoelastic and thermoelastic losses.

The first two terms are defined as [30]:

$$
\begin{aligned}
Q_{j, \text { air }}^{-1} & =\frac{R}{2 \pi \mu \nu_{j}}, R=2 \pi \eta_{\mathrm{air}}+2 \pi d \sqrt{\pi \eta_{\mathrm{air}} \rho_{\mathrm{air}} \nu_{j}}, \\
Q_{j, \mathrm{ve}}^{-1} & =\frac{4 \pi^{2} \mu E I \delta_{\mathrm{ve}}}{T^{2}} \nu_{j}^{2} .
\end{aligned}
$$

In these expressions, $\eta_{\text {air }}$ and $\rho_{\text {air }}$ are, respectively, the air dynamic viscosity coefficient and density. In the rest of the paper, usual values are chosen [49]: $\eta_{\text {air }}=1.8 \times 10^{-5} \mathrm{~kg} \mathrm{~m}^{-1} \mathrm{~s}^{-1}$ and $\rho_{\text {air }}=1.2 \mathrm{~kg}$ $\mathrm{m}^{-3}$. To complete the model, two parameters remain to be defined: the viscoelastic loss angle $\delta_{\mathrm{ve}}$, and the constant value $Q_{\mathrm{te}}^{-1}$ characterising the thermoelastic behaviour. As shown later in Section 4.2 (see also [30, 49]), these values can be fitted from experiments in order to finely model the frequency dependence of a real isolated string.

\subsubsection{Damping in the contact}

The model presented here may be complemented by nonlinear losses due to the contact, as described e.g. in [50, 44, 11, 45]. To this end, the contact force given by (3) may be augmented according to the Hunt and Crossley model [50]:

$$
f=\frac{\mathrm{d} \psi}{\mathrm{d} \eta}-\frac{\partial u}{\partial t} K \beta[\eta]_{+}^{\alpha}
$$

with $\beta \geq 0$.

\subsection{Spatial discretisation}

To circumvent the difficulty associated with the expression of the contact force with modal coordinates, a spatial grid is introduced, together with a linear relationship between modal coordinates and the displacement at points in the grid. The grid is defined as $x_{i}=i \Delta x$, where $\Delta x=\frac{L}{N}$ is the spatial step and $i \in\{0, \ldots, N\}$. Since $u\left(x_{0}, t\right)=0$ and $u\left(x_{N}, t\right)=0 \forall t \in \mathbb{R}^{+}$, only the values of $u$ on the grid with $i \in\{1,2, \ldots, N-1\}$ are examined in the following.

In the remainder of the paper we select $N_{m}=N-1$ such that the number of interior grid points will match the number of modes in the truncation. Then the modal expansion of $u(x, t)$ can be written at each point $i \in\{1,2, \ldots, N-1\}$ of the selected grid as:

$$
u\left(x_{i}, t\right)=u_{i}(t)=\sum_{j=1}^{N-1} q_{j}(t) \phi_{j}\left(x_{i}\right)=\sum_{j=1}^{N-1} q_{j}(t) \sqrt{\frac{2}{L}} \sin \left(\frac{j \pi i}{N}\right) .
$$

In matrix form, these relationships may be written as $\mathbf{u}=\mathbf{S q}$, where the vectors $\mathbf{u}=\left[u_{1}, \ldots, u_{N-1}\right]^{T}$ and $\mathbf{q}=\left[\begin{array}{lll}q_{1}, \ldots, & q_{N-1}\end{array}\right]^{T}$ have been introduced. The matrix $\mathbf{S}$ has entries $S_{i j}=\phi_{j}\left(x_{i}\right), \forall(i, j) \in$ $\{1, \ldots, N-1\}^{2}$, and its inverse satisfies the following relationship: $\mathbf{S}^{-1}=\frac{L}{N} \mathbf{S}^{T}$.

\subsection{Time discretisation}

Let $\mathbf{u}^{n}$ represent an approximation to $\mathbf{u}(t)$ at $t=n \Delta t$, for integer $n$, and where $\Delta t$ is a time step, assumed constant.

Difference operators may be defined as follows:

$$
\begin{aligned}
\delta_{t-} \mathbf{u}^{n} & =\frac{\mathbf{u}^{n}-\mathbf{u}^{n-1}}{\Delta t}, \quad \delta_{t+} \mathbf{u}^{n}=\frac{\mathbf{u}^{n+1}-\mathbf{u}^{n}}{\Delta t}, \quad \delta_{t .} \mathbf{u}^{n}=\frac{\mathbf{u}^{n+1}-\mathbf{u}^{n-1}}{2 \Delta t} \\
\delta_{t t} \mathbf{u}^{n} & =\frac{\mathbf{u}^{n+1}-2 \mathbf{u}^{n}+\mathbf{u}^{n-1}}{\Delta t^{2}} .
\end{aligned}
$$


The temporal scheme for discretising the equations of motion considers separately the modal linear part and the nonlinear contact force. For the linear part (the left-hand side of Eq. (11)), an exact scheme is proposed in [46] for a single oscillator equation. This scheme is here generalised to an arbitrary number $N-1$ of oscillator equations. This choice is justified by the accurate description of the frequency content and the stability property of this exact scheme, which perfectly recovers the oscillation frequencies irrespective of the time step. For the contact force, in order to circumvent the difficulty linked to the modal couplings in the right-hand side of Eq. (11), the relationship between $\mathbf{u}$ and $\mathbf{q}$ given in the previous section is used in order to treat the contact in the space domain.

The temporal scheme for the oscillatory part of Eq. (11) may be written as:

$$
\frac{\mu}{\Delta t^{2}}\left(\mathbf{q}^{n+1}-\mathbf{C q}^{n}+\tilde{\mathbf{C}} \mathbf{q}^{n-1}\right)=\mathbf{0}
$$

where the right-hand side has been neglected for the moment. $\mathbf{C}$ and $\tilde{\mathbf{C}}$ are diagonal matrices with entries

$$
\begin{aligned}
& C_{i i}=\mathrm{e}^{-\sigma_{i} \Delta t}\left(\mathrm{e}^{\sqrt{\sigma_{i}^{2}-\omega_{i}^{2}} \Delta t}+\mathrm{e}^{-\sqrt{\sigma_{i}^{2}-\omega_{i}^{2}} \Delta t}\right), \\
& \tilde{C}_{i i}=\mathrm{e}^{-2 \sigma_{i} \Delta t} .
\end{aligned}
$$

If there is no collision, and thus no contact force, so that the right-hand side equals zero, then the scheme is known to be exact [46, thus ensuring the most accurate discrete evaluation of the linear part. To determine the contact force, Eq. (11) is rewritten for the vector $\mathbf{u}$ thanks to the relationship $\mathbf{u}=\mathbf{S q}$ :

$$
\frac{\mu}{\Delta t^{2}}\left(\mathbf{u}^{n+1}-\mathbf{D} \mathbf{u}^{n}+\tilde{\mathbf{D}} \mathbf{u}^{n-1}\right)=\mathbf{f}^{n},
$$

with $\mathbf{D}=\mathbf{S C S}^{-1}$ and $\tilde{\mathbf{D}}=\mathbf{S} \tilde{\mathbf{C}} \mathbf{S}^{-1}$.

Following [11], the discrete approximation of the contact force is chosen as: $\mathbf{f}^{n}=\frac{\delta_{t-} \boldsymbol{\psi}^{n+\frac{1}{2}}}{\delta_{t} \cdot \boldsymbol{\eta}^{n}}$, where $\boldsymbol{\psi}^{n+\frac{1}{2}}=\frac{1}{2}\left(\boldsymbol{\psi}^{n+1}+\boldsymbol{\psi}^{n}\right)$ and $\boldsymbol{\psi}^{n}=\psi\left(\boldsymbol{\eta}^{n}\right)$. The resulting scheme is conservative if there is no loss, and dissipative otherwise (see Section 2.6).

The nonlinear equation to be solved at each time step is thus:

$$
\mathbf{r}+\mathbf{b}+m \frac{\psi(\mathbf{r}+\mathbf{a})-\psi(\mathbf{a})}{\mathbf{r}}=\mathbf{0},
$$

where $\mathbf{r}=\boldsymbol{\eta}^{n+1}-\boldsymbol{\eta}^{n-1}$ is the unknown (with $\left.\boldsymbol{\eta}^{n}=\mathbf{g}-\mathbf{u}^{n}\right), \mathbf{a}=\boldsymbol{\eta}^{n-1}, m=\frac{\Delta t^{2}}{\mu}$ and $\mathbf{b}=\mathbf{D} \mathbf{u}^{n}-(\tilde{\mathbf{D}}+$ $\left.\mathbf{I}_{N-1}\right) \mathbf{u}^{n-1}$. The Newton-Raphson algorithm may be used to this end. This equation has a unique solution [11, note however that the convergence of the Newton-Raphson algorithm is not guaranteed and depends on the initial guess. Note also that in the specific case of a linear restoring force (i.e. $\alpha=1$ ), an analytical solution is available as detailed in [51].

The additional damping term $\frac{\mathrm{d} u}{\mathrm{~d} t} K \beta[\eta]_{+}^{\alpha}$ due to collisions (see Section 2.3.2 may be discretised as follows [11]: $\delta_{t} \cdot \mathbf{u}^{n} K \beta\left[\boldsymbol{\eta}^{n}\right]_{+}^{\alpha}$. Instead of (17), the equation to be solved at each time step is then [11]:

$$
\left(\mathbf{I}_{N-1}+\mathbf{L}\right) \mathbf{r}+\mathbf{b}+m \frac{\psi(\mathbf{r}+\mathbf{a})-\psi(\mathbf{a})}{\mathbf{r}}=\mathbf{0},
$$

where $\mathbf{L}$ is a diagonal matrix such that $L_{i i}=\frac{\Delta t}{2 \mu} K \beta\left[\eta_{i}{ }^{n}\right]_{+}^{\alpha}$.

\subsection{Stability analysis}

This section is devoted to the stability analysis of the numerical scheme. To this end, it is more convenient to rewrite (16) with an explicit use of temporal discrete operators.

The equivalent representation may be written as:

$$
\mu\left[\check{\mathbf{C}}_{\mathbf{1}} \delta_{t t} \mathbf{q}^{n}+\check{\mathbf{C}}_{\mathbf{2}} \mathbf{q}^{n}+\check{\mathbf{C}}_{\mathbf{3}} \delta_{t} \cdot \mathbf{q}^{n}\right]=\mathbf{F}^{n},
$$


with diagonal matrices $\check{\mathbf{C}}_{\mathbf{1}}, \check{\mathbf{C}}_{\mathbf{2}}$ and $\check{\mathbf{C}}_{\boldsymbol{3}}$ with the following entries:

$$
\begin{aligned}
& \check{C}_{1_{i i}}=\frac{1+\left(1-\gamma_{i}\right) \frac{\omega_{i}^{2} \Delta t^{2}}{2}}{1+\left(1-\gamma_{i}\right) \frac{\omega_{i}^{2} \Delta t^{2}}{2}+\sigma_{i}^{*} \Delta t}, \\
& \check{C}_{2_{i i}}=\frac{\omega_{i}^{2}}{1+\left(1-\gamma_{i}\right) \frac{\omega_{i}^{2} \Delta t^{2}}{2}+\sigma_{i}^{*} \Delta t}, \\
& \check{C}_{3_{i i}}=\frac{2 \sigma_{i}^{*}}{1+\left(1-\gamma_{i}\right) \frac{\omega_{i}^{2} \Delta t^{2}}{2}+\sigma_{i}^{*} \Delta t} .
\end{aligned}
$$

The coefficients $\gamma_{i}$ and $\sigma_{i}^{*}$ may be written as:

$$
\begin{aligned}
\gamma_{i} & =\frac{2}{\omega_{i}^{2} \Delta t^{2}}-\frac{A_{i}}{1+e_{i}-A_{i}}, \\
\sigma_{i}^{*} & =\left(\frac{1}{\Delta t}+\frac{\omega_{i}^{2} \Delta t}{2}-\gamma_{i} \frac{\omega_{i}^{2} \Delta t}{2}\right) \frac{1-e_{i}}{1+e_{i}}
\end{aligned}
$$

where

$$
A_{i}=\mathrm{e}^{-\sigma_{i} \Delta t}\left(\mathrm{e}^{\sqrt{\sigma_{i}^{2}-\omega_{i}^{2}} \Delta t}+\mathrm{e}^{-\sqrt{\sigma_{i}^{2}-\omega_{i}^{2}} \Delta t}\right) \text { and } e_{i}=\mathrm{e}^{-2 \sigma_{i} \Delta t} .
$$

The equivalent scheme for the displacement $\mathbf{u}$ may thus be written as:

$$
\mu\left[\check{\mathbf{D}}_{\mathbf{1}} \delta_{t t} \mathbf{u}^{n}+\check{\mathbf{D}}_{\mathbf{2}} \mathbf{u}^{n}+\check{\mathbf{D}}_{\mathbf{3}} \delta_{t .} \mathbf{u}^{n}\right]=\mathbf{f}^{n},
$$

where $\check{\mathbf{D}}_{1}=\mathbf{S} \check{\mathbf{C}}_{1} \mathbf{S}^{-1}, \check{\mathbf{D}}_{2}=\mathbf{S} \check{\mathbf{C}}_{2} \mathbf{S}^{-1}$ and $\check{\mathbf{D}}_{3}=\mathbf{S} \check{\mathbf{C}}_{3} \mathbf{S}^{-1}$ are symmetric matrices. The force term is expressed as in Section 2.5 .

Let us introduce the inner product as:

$$
\langle\mathbf{u}, \mathbf{v}\rangle=\Delta x \sum_{j=1}^{N-1} u_{j} v_{j}
$$

where $\Delta x$ is the spatial step.

Taking the inner product between equation (21) and $\delta_{t} \cdot \mathbf{u}^{n}$, the following discrete energy balance is obtained:

$$
\delta_{t-} H^{n+\frac{1}{2}}=-\mu\left\langle\delta_{t} \cdot \mathbf{u}^{n}, \check{\mathbf{D}}_{\mathbf{3}} \delta_{t} \cdot \mathbf{u}^{n}\right\rangle
$$

where

$$
H^{n+\frac{1}{2}}=\frac{\mu}{2}\left\langle\delta_{t+} \mathbf{u}^{n}, \check{\mathbf{D}}_{\mathbf{1}} \delta_{t+} \mathbf{u}^{n}\right\rangle+\frac{\mu}{2}\left\langle\mathbf{u}^{n+1}, \check{\mathbf{D}}_{\mathbf{2}} \mathbf{u}^{n}\right\rangle+\left\langle\psi^{n+\frac{1}{2}}, 1\right\rangle .
$$

Because $\check{\mathbf{D}}_{\mathbf{3}}$ is positive semi-definite (see the proof in AppendixA Property 3 , the scheme is thus strictly dissipative. Therefore it is stable if the energy is positive.

The force potential being positive, and given Properties 1 and 3 demonstrated in AppendixA the stability condition can be rewritten as:

$$
\left\langle\delta_{t+} \mathbf{u}^{n},\left(\check{\mathbf{D}}_{1}-\frac{\Delta t^{2}}{4} \check{\mathbf{D}}_{\mathbf{2}}\right) \delta_{t+} \mathbf{u}^{n}\right\rangle \geq 0
$$

It is therefore sufficient to show that $\left(\check{\mathbf{D}}_{\mathbf{1}}-\frac{\Delta t^{2}}{4} \check{\mathbf{D}}_{\mathbf{2}}\right)$ is positive semi-definite to obtain stability, which is true if $\left(\check{\mathbf{C}}_{\mathbf{1}}-\frac{\Delta t^{2}}{4} \check{\mathbf{C}}_{\mathbf{2}}\right)$ is positive semi-definite. Consequently, the sufficient condition reads, $\forall i \in$ $\{1, \ldots, N-1\}$ :

$$
\gamma_{i} \leq \frac{1}{2}+\frac{2}{\omega_{i}^{2} \Delta t^{2}} .
$$

After a straightforward manipulation, the condition is easily expressed as:

$$
\frac{1+e_{i}+A_{i}}{1+e_{i}-A_{i}} \geq 0, \forall i \in\{1, \ldots, N-1\} .
$$


Eq. (26) is satisfied if $1+e_{i} \pm A_{i}>0$, which is always true (see Property 2 in AppendixA for the proof), and hence the scheme is unconditionally stable. The limiting case $\sigma_{i}=0$ corresponds to the lossless string. Then $\gamma_{i}$ reduces to:

$$
\gamma_{i}=\frac{2}{\omega_{i}^{2} \Delta t^{2}}-\frac{\cos \left(\omega_{i} \Delta t\right)}{1-\cos \left(\omega_{i} \Delta t\right)}
$$

and unconditional stability is obtained, as in the lossy case.

Considering contact losses (see Eq. (18) leads to the following discrete energy balance:

$$
\delta_{t-} H^{n+\frac{1}{2}}=-\mu\left\langle\delta_{t} . \mathbf{u}^{n}, \check{\mathbf{D}}_{\mathbf{3}} \delta_{t .} \mathbf{u}^{n}\right\rangle-\left\langle\delta_{t} . \mathbf{u}^{n}, \delta_{t} . \mathbf{u}^{n} K \beta\left[\boldsymbol{\eta}^{n}\right]_{+}^{\alpha}\right\rangle
$$

Since $\left\langle\delta_{t} . \mathbf{u}^{n}, \delta_{t} . \mathbf{u}^{n} K \beta\left[\boldsymbol{\eta}^{n}\right]_{+}^{\alpha}\right\rangle \geq 0$, the dissipation in the system is then increased, and the stability condition is not affected.

\subsection{Second polarisation}

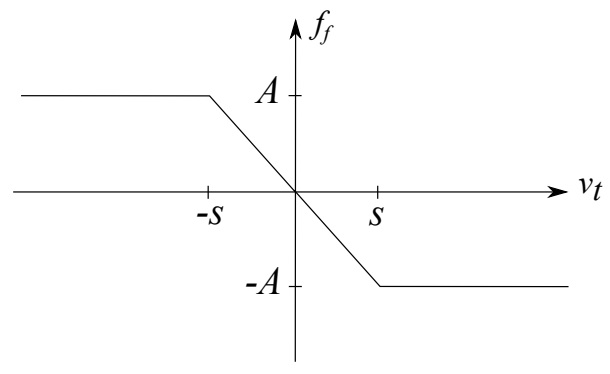

Figure 2: Friction force

In this section, the model is extended to include motion in the second polarisation of the string. The two unknown displacements along $(O z)$ and $(O y)$ are respectively denoted as $u(x, t)$ and $v(x, t)$. Assuming small displacements, the equations of motion for $u$ and $v$ are assumed uncoupled as long as no contact arises. In particular, no coupling is included at boundaries. As soon as a contact point is detected for the vertical displacement $u$, it is assumed that the horizontal displacement $v$ undergoes a friction force $f_{f}$. The continuous equation for the displacement $v$ may be written as:

$$
\mu v_{t t}-T v_{x x}+E I v_{x x x x}=\delta_{x_{c}}(x) f_{f}
$$

where $\delta_{x_{c}}$ is a Dirac delta function centered at $x_{c}$. Since the obstacle is assumed to be located at a point along $(O x)$ in this study, contact is assumed to arise at the location of the point obstacle $x_{c}$ only; it could, however, be extended to a larger contact surface. $f_{f}$ is a simple regularised Coulomb friction law defined as (see Fig. 22):

$$
f_{f}\left(v_{t}\right)=A\left\{\begin{array}{l}
1 \quad \text { if } v_{t}<-s \text { and } u<g \\
v_{t} / s \quad \text { if }\left|v_{t}\right| \leq s \text { and } u<g \\
-1 \quad \text { if } v_{t}>s \text { and } u<g \\
0 \quad \text { if } u \geq g,
\end{array}\right.
$$

where $v_{t}$ is the velocity of the string, and $A(\mathrm{~N}), s>0\left(\mathrm{~m} . \mathrm{s}^{-1}\right)$ are the two constant parameters that define the friction law. In particular, as shown in Section 5.2.3 these values can be fitted from experiments.

The expression for the stored energy associated with 28 is given by:

$$
\tilde{\mathscr{H}}=\frac{\mu}{2} \int_{0}^{L}\left(v_{t}\right)^{2} d x+\frac{T}{2} \int_{0}^{L}\left(v_{x}\right)^{2} d x+\frac{E I}{2} \int_{0}^{L}\left(v_{x x}\right)^{2} d x \geq 0,
$$

and satisfies

$$
\frac{d \tilde{\mathscr{H}}}{d t}=\tilde{\mathscr{Q}}, \text { where } \tilde{\mathscr{Q}}=v_{t}\left(x_{c}\right) f_{f}\left(v_{t}\left(x_{c}\right)\right)
$$


Applying the same method as for the contact between a string and a bow described in [46] and using a first order interpolation operator, one obtains:

$$
\mathbf{v}^{n+1}-\mathbf{D} \mathbf{v}^{n}+\tilde{\mathbf{D}} \mathbf{v}^{n-1}=\frac{\Delta t^{2}}{\mu} \mathbf{J}\left(x_{c}\right) f_{f}\left(\xi^{n}\right),
$$

where $\mathbf{J}\left(x_{c}\right)$ is a vector consisting of zeros except at the obstacle position $x_{c}$ where its value is $1 / \Delta x$. $\xi^{n}=\delta_{t} . v_{c}^{n}$ is the velocity of the string point interacting with the obstacle, which is the solution of the following equation:

$$
\left(-\mathbf{D v}^{n}+\left(\tilde{\mathbf{D}}+\mathbf{I}_{N-1}\right) \mathbf{v}^{n-1}\right)_{c}+2 \Delta t \xi^{n}-\frac{\Delta t^{2}}{\mu \Delta x} f_{f}\left(\xi^{n}\right)=0,
$$

where the subscript $c$ designates the element corresponding to the obstacle position. This equation depends on $\mathbf{u}^{n}$ through the force term $f_{f}$, see Eq. 29p. The discrete energy may be written as:

$$
\tilde{H}^{n+\frac{1}{2}}=\frac{\mu}{2}\left\langle\delta_{t+} \mathbf{v}^{n}, \check{\mathbf{D}}_{\mathbf{1}} \delta_{t+} \mathbf{v}^{n}\right\rangle+\frac{\mu}{2}\left\langle\mathbf{v}^{n+1}, \check{\mathbf{D}}_{\mathbf{2}} \mathbf{v}^{n}\right\rangle .
$$

It satisfies:

$$
\delta_{t-} \tilde{H}^{n+\frac{1}{2}}=-\mu\left\langle\delta_{t} . \mathbf{v}^{n}, \check{\mathbf{D}}_{\mathbf{3}} \delta_{t} . \mathbf{v}^{n}\right\rangle+\delta_{t} \cdot v_{c}^{n} f_{f}\left(\delta_{t} . v_{c}^{n}\right) .
$$

Since $\delta_{t} . v^{n}$ and $f_{f}\left(\delta_{t} v^{n}\right)$ are of opposite signs, the scheme is again strictly dissipative.

\section{Validation test}

\subsection{Convergence study}

The necessity of oversampling to avoid aliasing and obtain trustworthy results is mentioned in [33], due to the nonlinear contact which generates high frequencies. In this part, a detailed study of convergence is presented in order to fix the sampling rate that will be used for simulations.

\begin{tabular}{|c|c|c|c|c|}
\hline$L$ & $d$ & $T$ & $\mu$ & $B$ \\
\hline $1.002 \mathrm{~m}$ & $0.43 \mathrm{~mm}$ & $180.5 \mathrm{~N}$ & $1.17 \times 10^{-3} \mathrm{~kg} \cdot \mathrm{m}^{-1}$ & $1.78 \times 10^{-5}$ \\
\hline
\end{tabular}

Table 1: Physical properties of the string

The particular string under study here is an electric guitar string manufactured by D'Addario, the properties of which are detailed in Table 1. Under a tension of $180.5 \mathrm{~N}$, it has a fundamental frequency of approximately $196 \mathrm{~Hz}$ (G3). The initial condition is set to a symmetric triangular shape of height $u_{0, \max }=1.8 \mathrm{~mm}$ with a smooth corner obtained by considering the 50 first modes, without initial velocity. The observed signal is taken at $10 \mathrm{~mm}$ from the extremity $x=L$. Simulations are conducted with $F_{s}$ from $1960 \mathrm{~Hz}$ to $2^{11} \times 1960 \mathrm{~Hz} \approx 4 \mathrm{MHz}$.

\begin{tabular}{|c|c|c|c|c|}
\hline \multicolumn{5}{|c|}{$N=2$} \\
\hline$\alpha / K$ & $10^{7}$ & $10^{9}$ & $10^{11}$ & $10^{13}$ \\
\hline 1 & $2.4 \times 10^{-5}$ & $2.4 \times 10^{-6}$ & $3.0 \times 10^{-7}$ & $3.4 \times 10^{-8}$ \\
1.5 & $2.2 \times 10^{-4}$ & $3.5 \times 10^{-5}$ & $5.5 \times 10^{-6}$ & $9.2 \times 10^{-7}$ \\
2 & $8.7 \times 10^{-4}$ & $2.0 \times 10^{-4}$ & $4.4 \times 10^{-5}$ & $9.5 \times 10^{-6}$ \\
\hline \multicolumn{5}{|c|}{$N=1002$, centered point obstacle } \\
\hline$\alpha / K$ & $10^{7}$ & $10^{9}$ & $10^{11}$ & $10^{13}$ \\
\hline 1 & $2.1 \times 10^{-4}$ & $2.3 \times 10^{-6}$ & $1.9 \times 10^{-7}$ & $1.8 \times 10^{-8}$ \\
1.5 & $1.4 \times 10^{-3}$ & $1.5 \times 10^{-4}$ & $7.6 \times 10^{-6}$ & $6.7 \times 10^{-7}$ \\
2 & $1.8 \times 10^{-3}$ & $1.1 \times 10^{-3}$ & $1.3 \times 10^{-4}$ & $1.4 \times 10^{-5}$ \\
\hline
\end{tabular}

Table 2: Maximal penetration $(\mathrm{m}), F_{s}=2 \mathrm{MHz}, B=1.78 \times 10^{-5}$.

Convergence curves are presented in Fig. 3 for 1 and 1001 interior points (i.e. $N=2$ and $N=1002$ respectively), in the case of a centered point obstacle. The lossless and lossy string cases are presented for $N=2$, but only the latter is considered for $N=1002$. Computations are conducted over 3 seconds, 


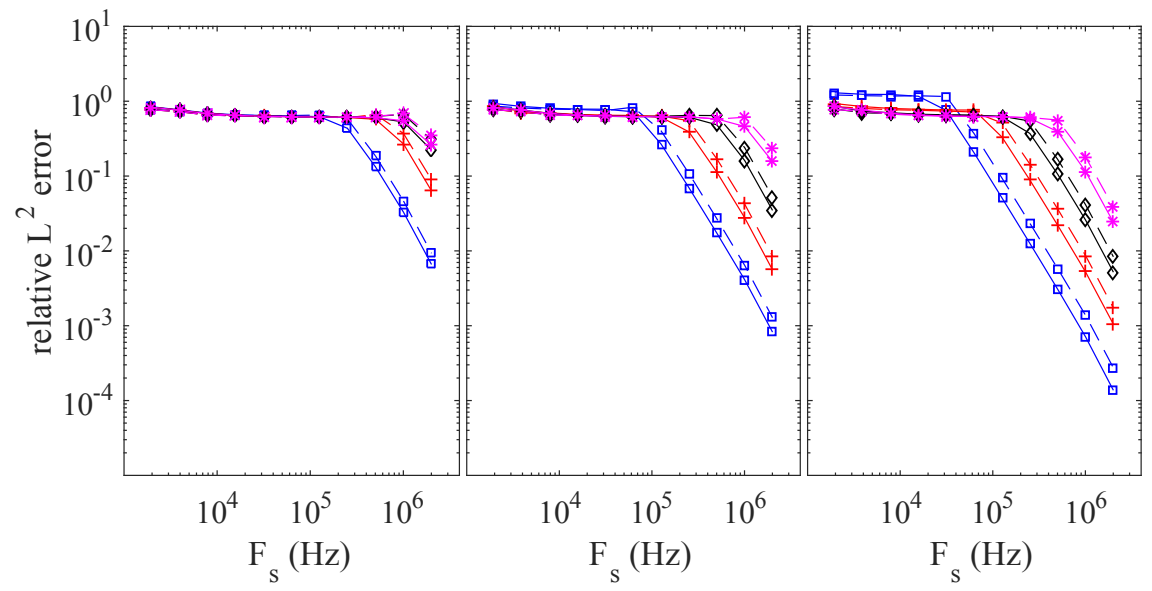

(a)

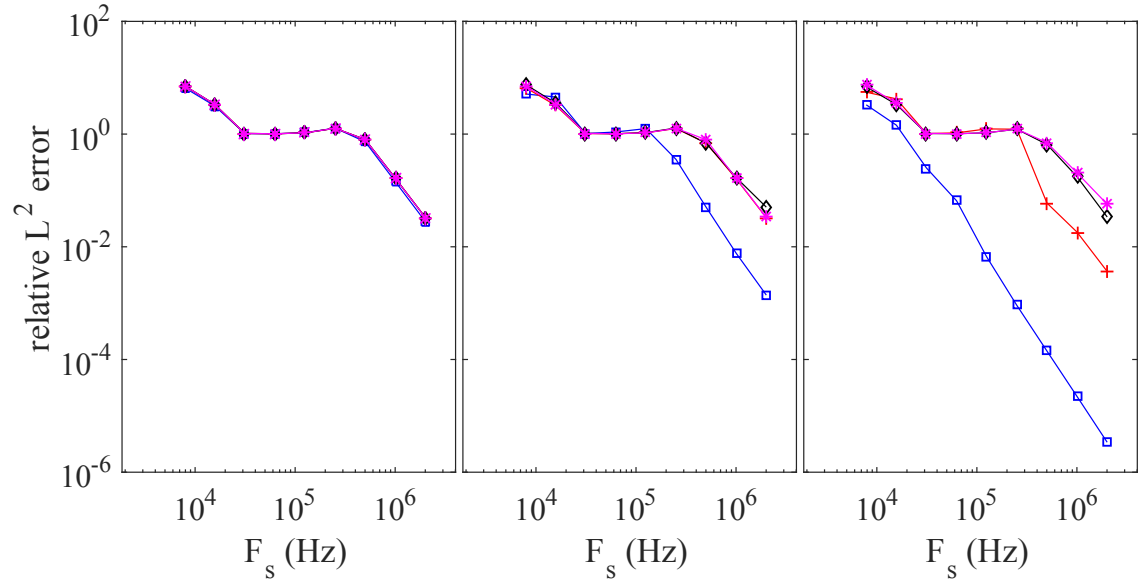

(b)

Figure 3: Relative $\mathrm{L}^{2}$ error versus $F_{s}$, over 3 seconds. Left: $\alpha=1$, centre: $\alpha=1.5$, right: $\alpha=2 . K=10^{7}$ (blue), $K=10^{9}$ (red), $K=10^{11}$ (black) and $K=10^{13}$ (magenta). (a) $N=2$, lossless (solid lines) and lossy (dashed lines) stiff string, $B=1.78 \times 10^{-5}(\mathrm{~b}) N=1002$, centered point obstacle, lossy stiff string, $B=1.78 \times 10^{-5}$.

corresponding to the duration used to compare experimental and numerical results in the following section. Convergence tests are thus more strict than for short durations. The relative $\mathrm{L}^{2}$ error is defined as:

$$
\left(\frac{\sum_{t \in \tau}\left(s_{r e f}(t)-s_{c u r}(t)\right)^{2}}{\sum_{t \in \tau}\left(s_{r e f}(t)\right)^{2}}\right)^{\frac{1}{2}},
$$

where $s_{\text {cur }}$ is the current signal with $F_{s}<4 \mathrm{MHz}$ and $s_{\text {ref }}$ the reference signal with $F_{s} \approx 4 \mathrm{MHz}$. Both are drawn from the string displacement at $10 \mathrm{~mm}$ from the boundary $x=L$. Sums are computed over the set $\tau$ of discrete times at which the signal having the lowest sampling rate (about $2 \mathrm{kHz}$ ) is evaluated. As is to be expected, the addition of losses leads to faster convergence. Also as is to be expected, the smoother the contact is (which corresponds to larger values of $\alpha$ and / or smaller values of $K$ ), the faster the convergence is, since less high frequency content is generated due to the contact.

The slope 2 corresponding to the order of the scheme is visible after a threshold sampling rate is reached. For the first sampling rates, in most of the cases presented here, a plateau can be observed. This may be due to the fact that for lower sampling rates, all the physical spectral content of the signal is not yet fully represented, so that the expected convergence speed cannot appear. This would explain that the stiffer the contact is, the larger the threshold sampling rate is, since higher frequencies are generated. In the case $N-1=1$, the first signals differ from the $4 \mathrm{MHz}$ signal mostly because of a phase difference which increases at each contact, as illustrated in Fig. 4. Table 2 details maximum penetration of the string in the obstacle for $F_{s}=2 \mathrm{MHz}$. The smoother the contact is, the larger the penetration is. A correlation can be made between convergence behaviour and maximum penetration, 


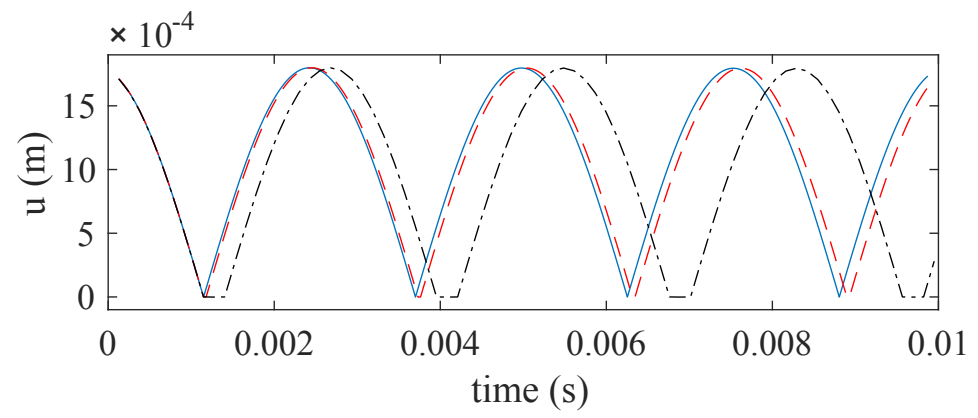

Figure 4: Displacement with $N-1=1, K=10^{13}, \alpha=1.5 . F_{s} \approx 8 \mathrm{kHz}$ (dark dash-dot line), $64 \mathrm{kHz}$ (red dashed line) and $4 \mathrm{MHz}$ (blue line).

since penetration is directly linked to stiffness of the contact and therefore to the amount of generated high frequencies. When penetration is greater than the string diameter, the relative error for $F_{s}=2$ $\mathrm{MHz}$ is smaller than $1 \times 10^{-2}$. For smaller penetration however, convergence is significantly slower.

In the context of our study, rigid contact is intended so that values $\alpha=1.5$ and $K=10^{13}$ are selected by empirical comparisons to experiments. In the case of a point obstacle, it appears that smoothing the contact makes numerical signals differ more from experimental ones, whereas making it stiffer does not significantly improve results. In order to conduct simulations, in the sequel, the sampling rate is chosen as corresponding to a relative L2 error smaller than about $1 \times 10^{-1}$. Then a sampling rate of at least $F_{s}=1 \mathrm{MHz}$ is necessary. For an extra degree of safety, $F_{s}=2 \mathrm{MHz}$ is selected in the following.

\begin{tabular}{|c|c|c|c|c|}
\hline$F_{s}$ & $N$ & $\alpha$ & $K$ & $\beta$ \\
\hline $2 \mathrm{MHz}$ & 1002 & 1.5 & $10^{13}$ & 0 \\
\hline
\end{tabular}

Table 3: Numerical parameters

\subsection{Comparison to the analytical solution}

The outcomes of the numerical scheme $(16)$ are first compared to an analytical solution presented by H. Cabannes [18, 52, for the case of an ideal string, with a centered point obstacle in contact with the string at rest. The analytical solution assumes contact with no interpenetration, corresponding to a perfectly rigid point obstacle. Consequently the numerical parameters for the contact law are selected as $\alpha=1.5$ and $K=10^{13}$. The initial condition in displacement has the shape of a triangle, with an initial velocity of zero everywhere. In order to facilitate comparisons with the analytical solution, results are dimensionless in the present section. To this end, dimensionless values are used in simulations. $N-1=1001$ interior points and a dimensionless sampling rate $F_{s, d}=5000$ (corresponding to a sampling rate of about $2 \mathrm{MHz}$, see Section 3.1) are selected. Subscript $d$ stands for "dimensionless". Stiffness and damping parameters are chosen as $B_{d}=2 \times 10^{-5}$ and $\sigma_{d, j}=j \times 5 \times 10^{-3}, \forall j \in$ $\{1, \ldots, N-1\}$.

For a qualitative comparison, Fig. 5 shows successive snapshots of the profile of the ideal string during its vibration. As already noted in [18], the contact is persistent and occurs as long as the string is under its rest position. With this view the numerical solution perfectly coincides with the analytical one.

A more quantitative comparison is shown in Fig. 6 by focusing on the very beginning of the motion. Also, in order to get a better understanding of the individual effects of damping and stiffness terms in the model, they are incorporated step by step to investigate how the solution departs from that of the ideal string. The time series shown in Fig. 6 represents the output $u\left(x_{m}, t\right)$, where the point $x_{m}$ is located at $9 L / 100$. Fig. 6.a) and (b), for an ideal string, respectively without and with obstacle, show that the numerical solution closely matches the analytical one. In particular, one can observe that the fundamental frequency in the case with obstacle is equal to the one without obstacle multiplied by the ratio $4 / 3$, as theoretically predicted [52]. Fig. 6(c) shows the effect of damping on the numerical simulation. Small unevennesses appears, specified by arrows in Fig. 6(c). They are most probably due to the rounding of traveling corners. Finally, adding a small stiffness value in the string creates 

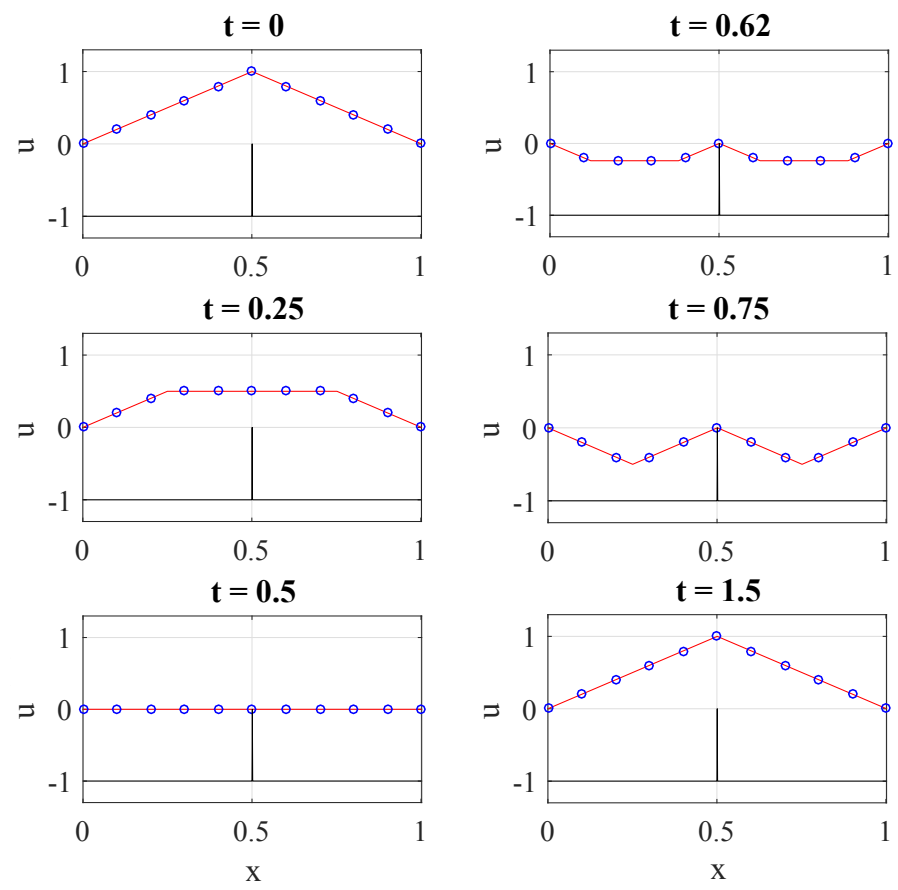

Figure 5: Snapshots of the motion of a dimensionless ideal string colliding with a point obstacle at centre (in black). Comparison at six different times of the first period between the analytical solution (blue circles) and the numerical one (modal approach, red line). Simulation conducted with $F_{s, d}=5000, K=10^{13}, \alpha=1.5$ and $\beta=0$. Presented variables are dimensionless.

dispersive waves, which in turn produce precursors since high frequencies arrive before lower ones at the measurement point. Previously mentioned unevennesses also appear in this case because stiffness causes rounding of corners.

Finally, energy variations of the numerical stiff string are presented in Fig. 7, with and without obstacle. As no damping is included in this numerical simulation, the energy is conserved: the normalised energy variations from one time step to the next are small, and of the order of $10^{-10}$. One can also observe that during the time interval where the contact occurs (indicated with a bold blue line in Fig. 7b, small oscillations in the contact energy appear which are due to very small oscillations of the string at the contact point. The behaviour of the string at this point will be further addressed in Section 5.2.2. Consistency of numerical results compared to the analytical solution has thus been highlighted, as well as effects of the string damping and stiffness. Energy considerations have also been presented. In the following, an experimental set up is presented, which will be exploited to compare numerical results against experiments.

\section{Experimental study}

\subsection{Experimental set up}

\subsubsection{Measurement frame}

The string to be considered here is described in Section 3.1. The vibration of this string, isolated from any surrounding structure, is studied on a measurement frame designed to this end [53, 49] (see Fig. 8, where two configurations are presented: the first with a centered obstacle, the second with an obstacle near a boundary). The string is plucked with a $0.05 \mathrm{~mm}$ diameter copper wire that breaks at the initial time [49] at the middle of the string. The maximal initial displacement is about $u_{0, \max }=1.5 \mathrm{~mm}$ in the rest of the paper. The vertical and horizontal displacements are measured with optical sensors described in [54. They are located near the string end at $x=L$, respectively at 1 $\mathrm{cm}$ (vertical) and $2 \mathrm{~cm}$ (horizontal). In the present study, the obstacle touches the string at rest. The point obstacle is realised with a metal cuboid edge. It is mounted on a vertical displacement system with a micron-scale sensitivity. 
(a)

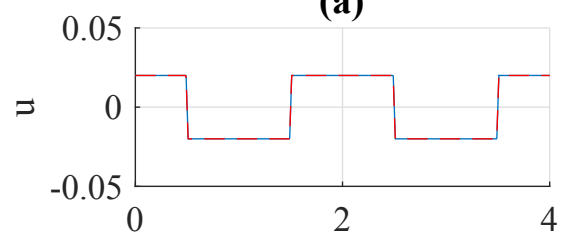

(c)

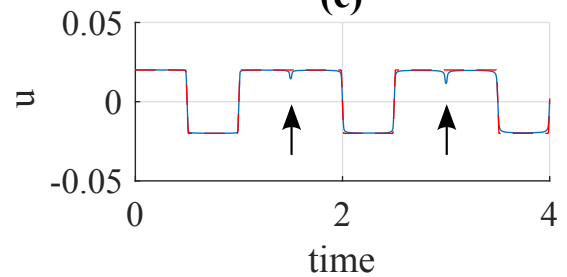

(b)

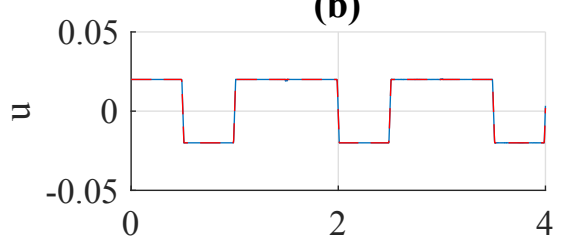

(d)

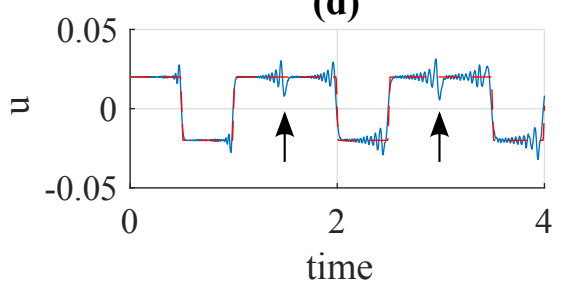

Figure 6: Time signal of the dimensionless string at $x_{m}=9 L / 100$, comparison of analytical solution for an ideal string (red dashed line), and numerical results (blue line). $N=1002, F_{s, d}=5000$. Variables are dimensionless. (a) ideal string without losses or dispersion, and without obstacle. (b) ideal string with obstacle. (c) losses added in the numerical simulation, with obstacle. (d) dispersive lossless numerical string with obstacle, $B_{d}=2 \times 10^{-5}$.

\subsubsection{Contact detection}

In order to detect contact between the string and the obstacle, an electrical circuit is installed on the measurement frame (see Fig. 9p. The switch links the string and the obstacle, which are both conductive. Voltage at its terminals is measured as an indicator of contact, being null when the string touches the obstacle. In order to avoid electric arcs for small distances between the string and the obstacle, components inside the acquisition card $\left(R_{N I}=300 \mathrm{k} \Omega\right.$ and $\left.C_{N I}=10.4 \mathrm{pF}\right)$ must be taken into account. A $10 \mathrm{kHz}$ alternative current has been employed and $R=100 \mathrm{k} \Omega$ has been chosen.

\subsection{Identification of linear characteristics}

In order to identify linear parameters of the string, i.e. eigenfrequencies and modal damping ratios, free vibrations of the string in the absence of the obstacle are measured and analysed with the ESPRIT method [55]. This method is applied to 4 seconds of the signal, starting 0.2 seconds after the string is plucked in order to avoid the transitory regime. Modes are treated one by one, according to the procedure described in [56, 49. The linear characteristics of 36 modes have been recovered with the method, which covers a frequency range up to $7200 \mathrm{~Hz}$. Beyond this frequency, modes are not excited strongly enough in the measured signals and the signal to noise ratio becomes too small to enable identification. In order to determine the remaining values, theoretical models are employed. The eigenfrequencies are then given by $\nu_{j}=j \frac{c_{0}}{2 L} \sqrt{1+B j^{2}}$ (see Section 2.2), where the inharmonicity factor $B$ (see Table 1) is determined by fitting the model to measurements. Damping parameters are obtained from the damping model presented in Section 2. This representation depends on two parameters, $\delta_{\mathrm{ve}}$ and $Q_{\mathrm{te}}^{-1}$, which are determined from experimental fitting. Selected values are $\delta_{\mathrm{ve}}=4.5 \times 10^{-3}$ and $Q_{\mathrm{te}}^{-1}=2.03 \times 10^{-4}$. These parameters will be used in the rest of the paper.

Measured values together with uncertainties (obtained over nine measurements, covering repeatability measurement errors and ESPRIT method uncertainties), theoretical model results and errors between them are shown in Fig. 10 and 11. One can observe that the inharmonicity of the string (and thus its stiffness) is very small. The damping model gives a very accurate representation of the measured losses. Uncertainties on frequencies are around $0.1 \%$, therefore not visible in Fig. 10. Errors in the frequencies and quality factors are respectively smaller than $0.2 \%$ and $25 \%$.

Finally, a highly controlled set up has been presented and linear parameters of the string have been accurately determined. Obtained parameters can therefore be employed in the numerical model described in Section 2 and a comparison between numerical and experimental results is possible.

\section{Numerical vs experimental results}

In this section, numerical and experimental signals are compared over long durations, in the time and frequency domains. Three cases are considered: the vibrating string without obstacle, or with a 


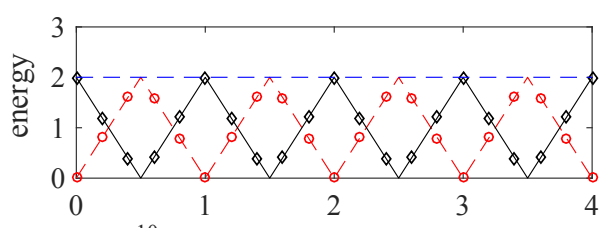

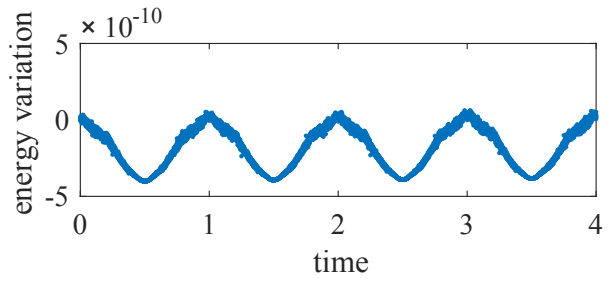

(a)
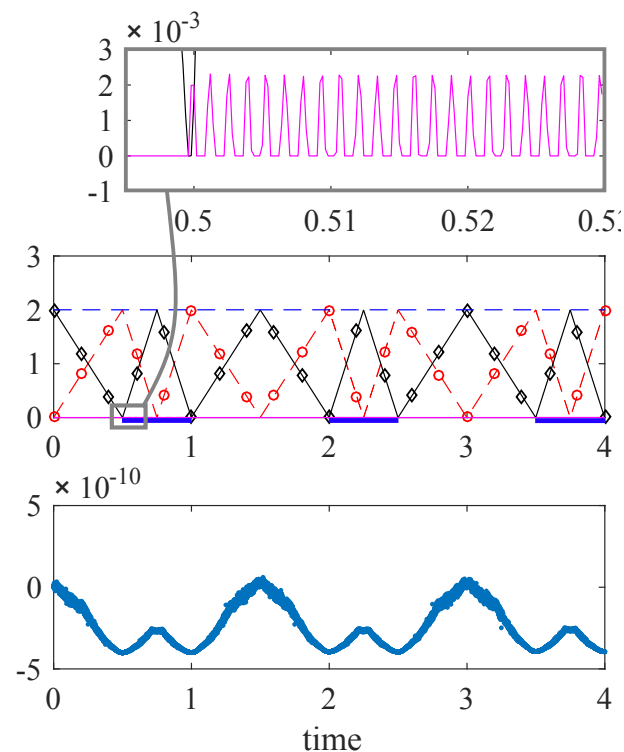

(b)

Figure 7: Energetic behaviour of the numerical ideal lossless vibrating string, $F_{s, d}=5000$. Variables are dimensionless. Top: energy of the numerical signal; kinetic energy (red dashed line and circles); potential energy (dark line and diamonds); total energy (blue dashed line). Bottom: relative energy variation $\frac{H^{n+1 / 2}-H^{1 / 2}}{H^{1 / 2}}$ (a) No obstacle. (b) Centered point obstacle. The contact energy (magenta line) is also presented. Bold blue lines indicate the time interval during which contact is persistent, resulting in an oscillatory pattern for the contact energy, shown in the upper inset.

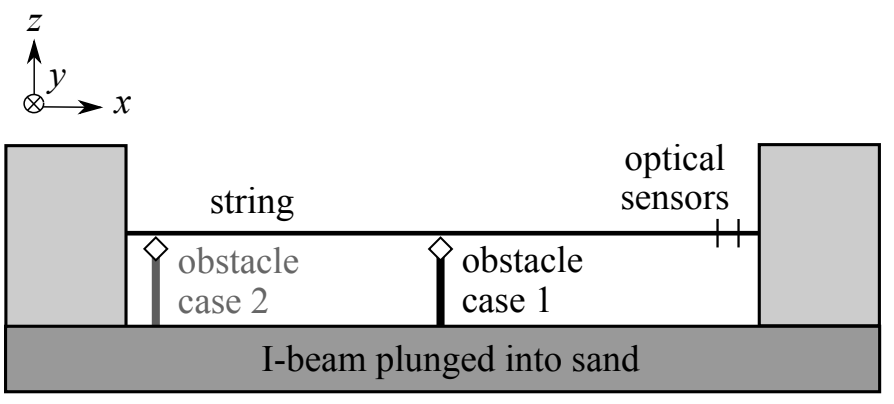

Figure 8: Schematic representation of the measurement frame.

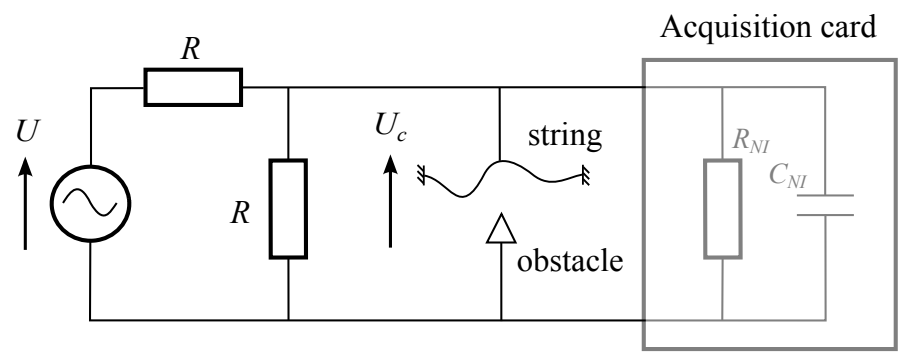

Figure 9: Electric scheme for contact detection.

point obstacle either centered or near a boundary, the latter constituting a two point bridge.

Selected string and numerical parameters are presented in Tables 1 and 3.

In all experimental results presented here except in Section 5.2.3 the initial condition is located in the $(x O z)$ plane, so that almost no initial energy is communicated to the horizontal polarisation. It has indeed been observed in all measurements that with this type of initial condition the horizontal oscillations were negligible. We thus focus on the vertical motion only in these cases. Associated 


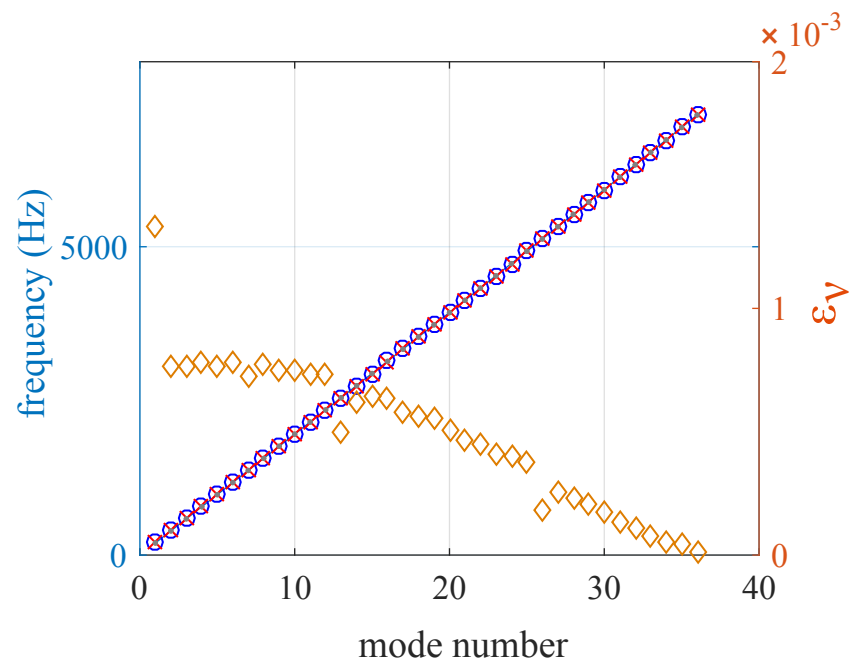

Figure 10: Experimental (red crosses) and theoretical (blue circles) eigenfrequencies, $\nu_{m}$ and $\nu_{t h}$ respectively, expanded uncertainty (gray lines) and error indicator (orange diamonds) $\epsilon_{\nu}=\frac{\left|\nu_{t h}-\nu_{m}\right|}{\nu_{m}}$, with $B=1.78 \times 10^{-5}$.

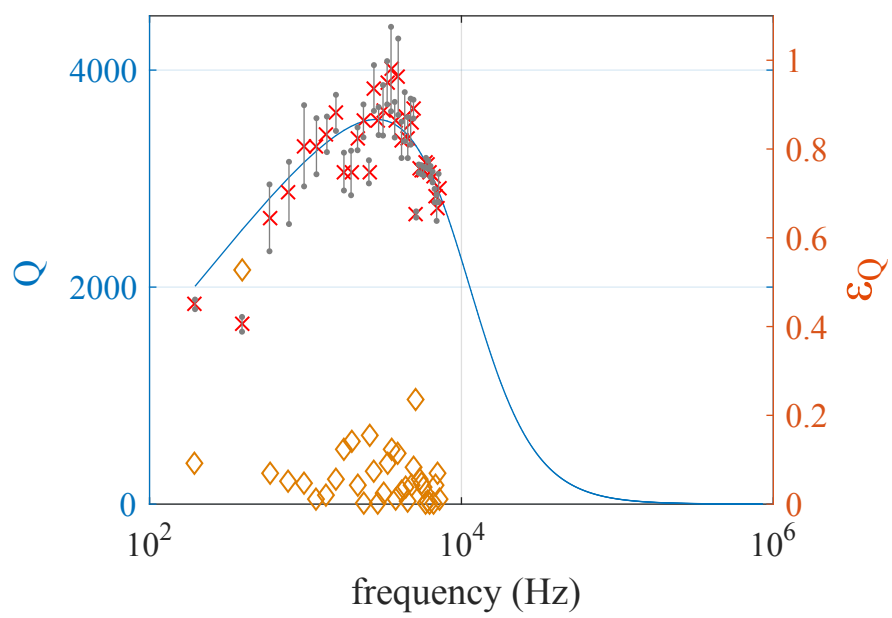

Figure 11: Experimental (red crosses) and theoretical (blue line) quality factors, $Q_{m}$ and $Q_{t h}$ respectively, expanded uncertainty at $95 \%$ (gray lines) and error indicator (orange diamonds) $\epsilon_{Q}=\frac{\left|Q_{t h}-Q_{m}\right|}{Q_{m}}$, with $\delta_{\mathrm{ve}}=4.5 \times 10^{-3}$ and $Q_{\mathrm{te}}^{-1}=2.03 \times 10^{-4}$.

sounds are available on the companion web-page of the paper 1 . They correspond to the displacement along $(O z)$ at $x=992 \mathrm{~mm}$, resampled at $44.1 \mathrm{kHz}$.

\subsection{No obstacle}

Fig. 12 shows the comparison between experimental and numerical results, when there is no obstacle, at the location of the optical sensor, i.e. at $1 \mathrm{~cm}$ from the edge $x=L$. The parameters of the numerical simulation are specified in Tables 1 and 3 . Dispersion effects are clearly visible in the first periods where the waveform is close to a rectangular function, then losses make the waveform evolve with the same progression numerically and experimentally. A minor error in the amplitude of the response is noticeable.

\footnotetext{
${ }^{1}$ Sounds are available in the companion web-page of the paper hosted by Elsevier as well as at http://www.lam.jussieu.fr/Membres/Issanchou/Sounds_vibrating_string_point_obstacle.html.
} 


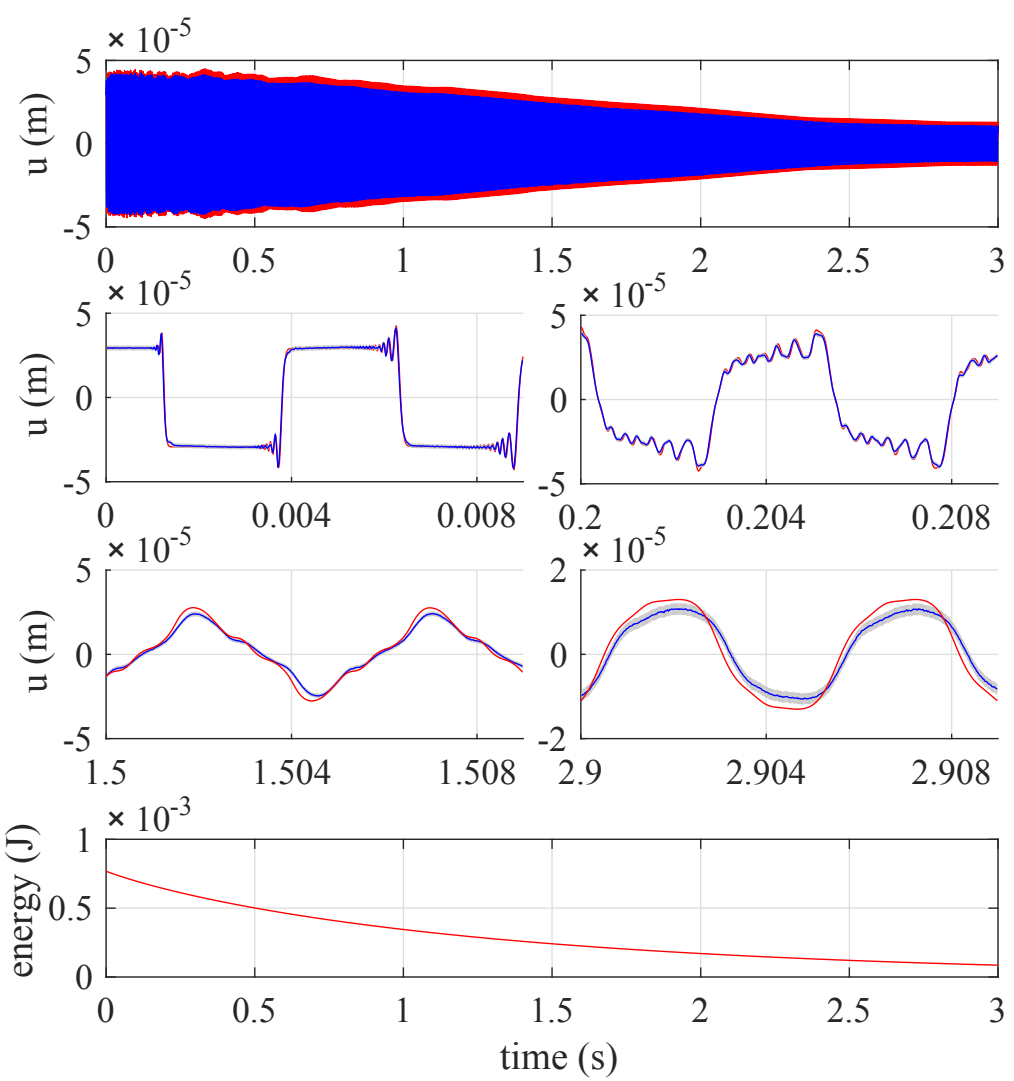

Figure 12: Displacement of the string when vibrating without obstacle, $B=1.78 \times 10^{-5}$. Comparison between measurement (blue line) and numerical simulation (red line), vertical displacement at $1 \mathrm{~cm}$ near the edge $x=L$. Expanded uncertainty at $95 \%$ (gray). Bottom shows the temporal decrease of the energy numerically computed.

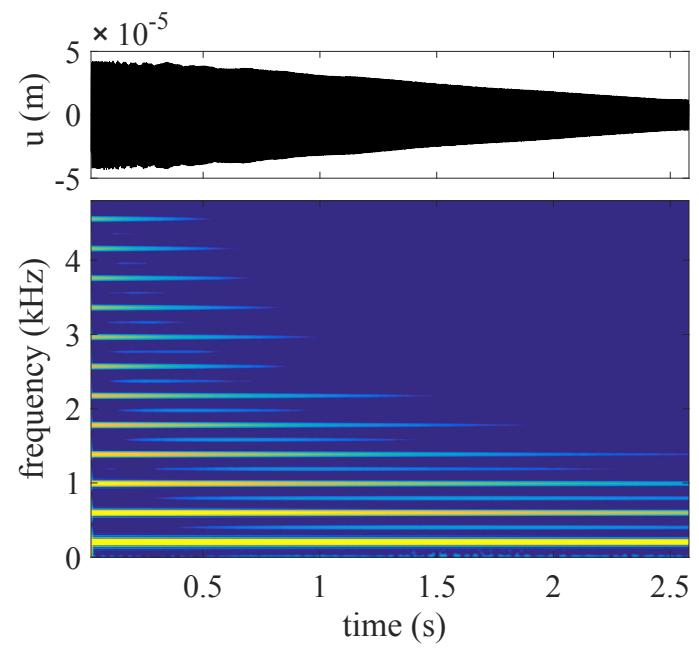

(a)

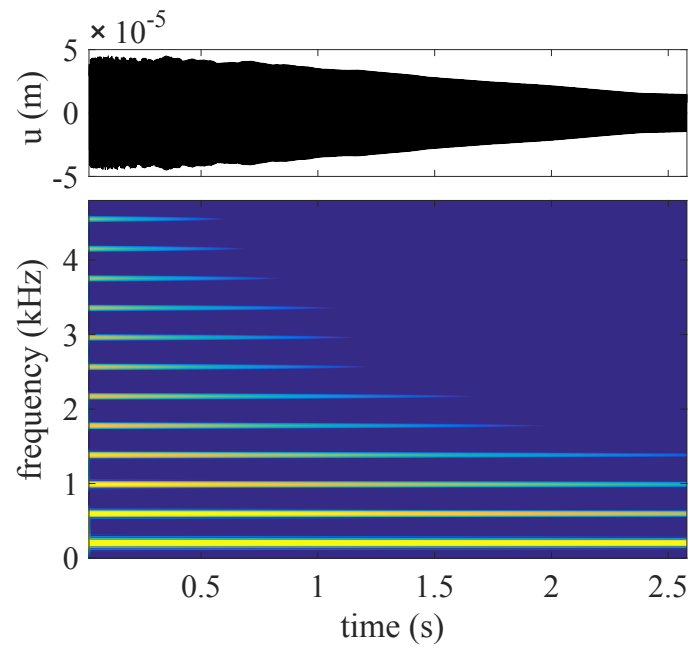

(b)

Figure 13: Vibrating string without obstacle, $B=1.78 \times 10^{-5}$. Spectrograms of the displacement: (a) experimental, (b) numerical.

The spectrograms of experimental and numerical signals are compared in Fig. 13, underlining the similarity of the frequency content of both time series. Due to the nature of the initial condition (even function with respect to the centre point), odd modes should not be excited. However one can observe the trace of these modes in the experimental spectrogram, even though their amplitudes are more than $60 \mathrm{~dB}$ below the amplitude of the first mode. This should be attributed to small imperfections in the string or boundary conditions, or to a small deviation of the experimental initial condition from the 
perfect symmetric triangle. In the simulation, the odd modes are completely absent. Finally, one can also observe that the damping of the upper modes seems to be slightly underestimated in the numerical simulation since their energy remains visible approximately $0.1 \mathrm{~s}$ longer on the spectrograms.

\subsection{Centered point obstacle}

In this section, the vibration of a string against a centered point obstacle is examined. First, the string is excited in the $(x O z)$ plane. The contact is investigated in detail and the second polarisation is observed.

\subsubsection{Temporal and spectral description}

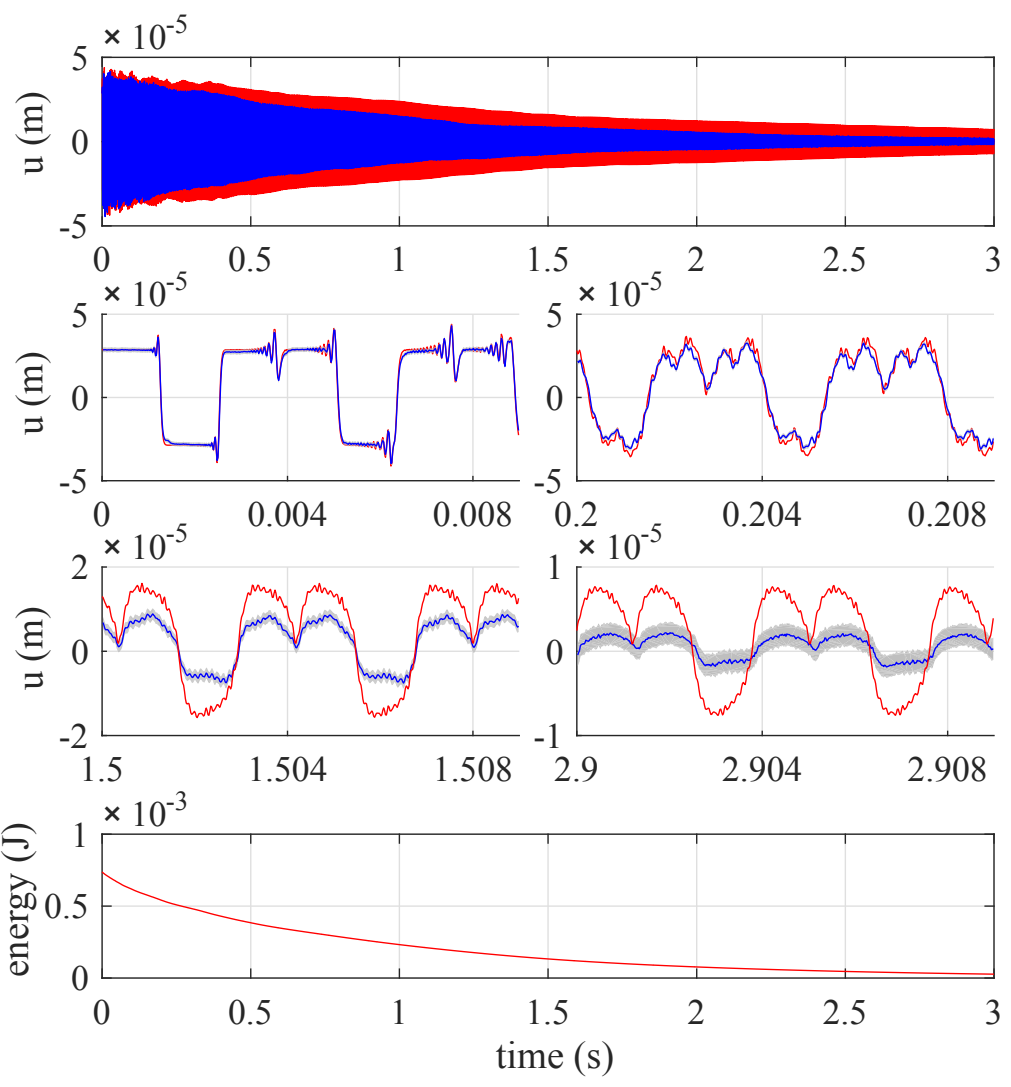

Figure 14: Displacement of the string when vibrating with a centered point obstacle, $B=1.78 \times 10^{-5}$. Comparison between measurement (blue line) and numerical simulation (red line), vertical displacement at $1 \mathrm{~cm}$ near the edge $x=L$. Expanded uncertainty at $95 \%$ (gray). Bottom shows the temporal decrease of the energy numerically computed.

Fig. 14 presents numerical and experimental signals in the case of a centered point obstacle and initial excitation along $(O z)$. As in Section 5.1 similarities can be observed, in the global shape of the signal as well as in its detailed behaviour. The ratio between numerical frequencies without obstacle $f_{1}$ and with the centered obstacle $f_{2}$ satisfies $\frac{f_{1}}{f_{2}} \approx \frac{195.7}{261.3} \approx \frac{3}{4}$, as expected from the theory (see Section 3.2 . Fine features of the experimental signal are reproduced numerically, as can be seen in enlarged views of the results. The dynamics including the contact is well-reproduced, and the numerical waveform evolves similarly to the experimental one. However, a significant error in amplitude appears, which may be due to uncertainty in the obstacle position and height, non-ideal experimental boundaries and initial conditions or dissipation as contact occurs. It could also be due to an imperfect rigid obstacle. Note that adding losses in the contact as described in Section 2.3.2 reduces the amplitude, so that the global shape better fits the experimental one. Nevertheless, this is at the cost of the local waveform shape, as illustrated in Fig. 15. Therefore no contact damping is included in the following (i.e. $\beta=0)$.

Spectrograms of experimental and numerical signals are presented in Fig. 16, they once again show strong similarities. Since modes are coupled through the contact, there is no missing mode, contrarily to the case without obstacle. A peculiar feature is a spectral resurgence zone around $8 \mathrm{kHz}$, underlined by a brace in Fig. 16b which clearly appears on both numerical and experimental spectrograms, 

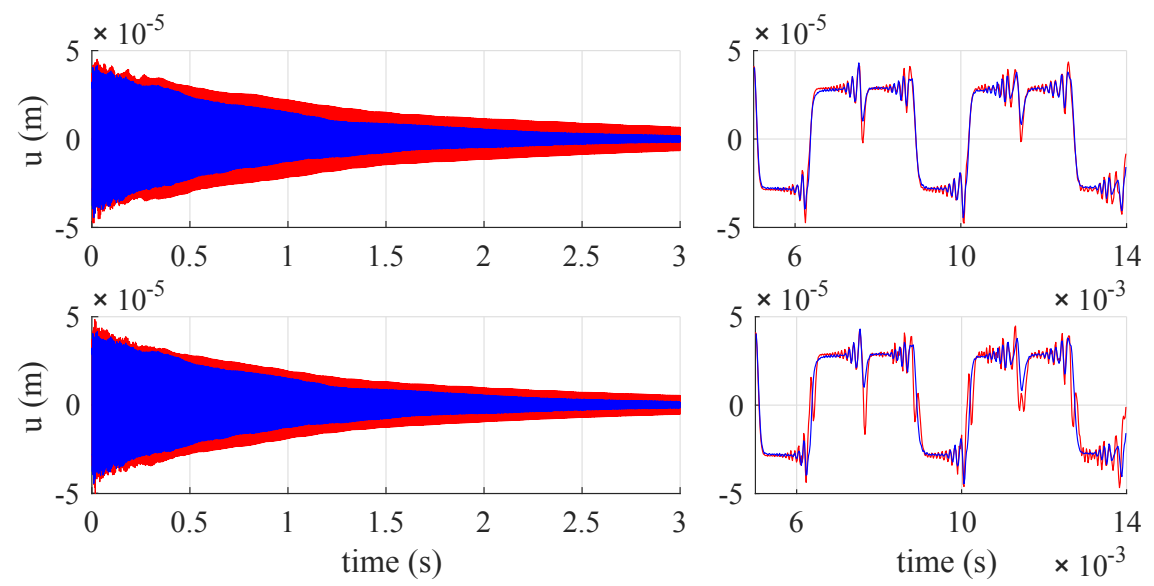

Figure 15: Numerical (red line) and experimental (blue line) displacement signals with a centered point obstacle, with $B=1.78 \times 10^{-5}$, including contact losses with $\beta=500$ (top) and $\beta=1000$ (bottom).

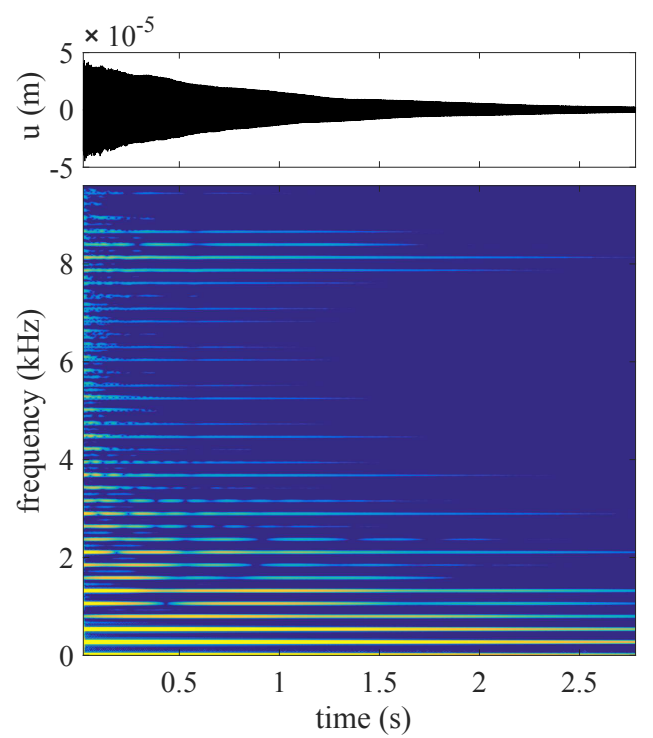

(a)

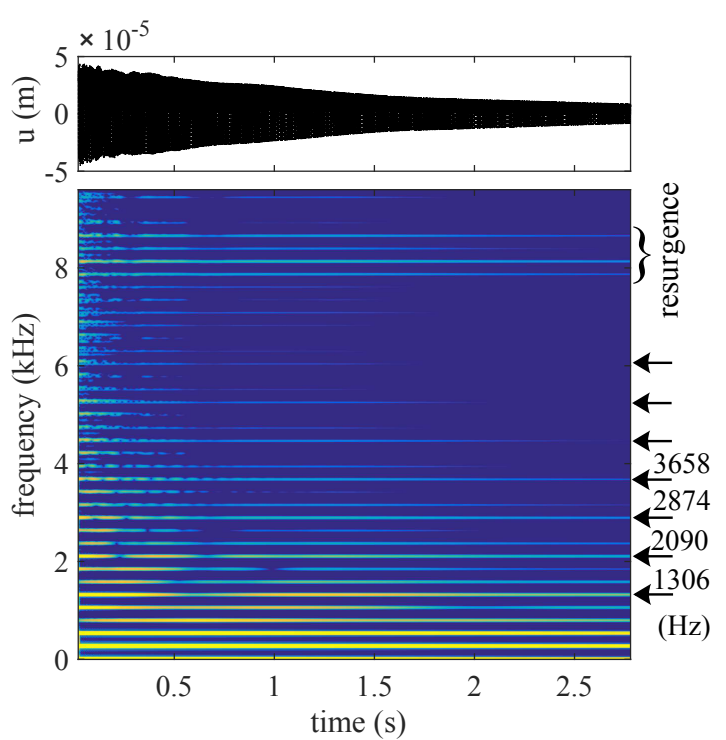

(b)

Figure 16: Centered obstacle, $B=1.78 \times 10^{-5}$. Spectrograms: (a) experimental, (b) numerical.

showing that energy can be transfered thanks to the contact up to these high frequencies. It is also a signature of the dispersion since cancelling the stiffness term makes this zone disappear. A second distinctive feature of the spectrograms is the appearance of spectral peaks with larger amplitudes, around $1306 \mathrm{~Hz}, 2090 \mathrm{~Hz}, 2874 \mathrm{~Hz}, 3658 \mathrm{~Hz}, \ldots$, see Fig. 16b where arrows indicate their presence. The difference between two of each of these successive peaks is equal to $784 \mathrm{~Hz}$, indicating that a rule governs their appearance. This value of $784 \mathrm{~Hz}$ is related to the ratio $3 / 4$ observed previously between the fundamental frequency of the string $(196 \mathrm{~Hz})$ and the fundamental frequency of the oscillations in the case with contact $(261 \mathrm{~Hz})$, since one has: $784=196 \times 4 \approx 261 \times 3$. Moreover, the dimensionless value of the period associated to $784 \mathrm{~Hz}$ is equal to 0.5 . Returning back to Fig. 6 one can observe that in Fig. 6(c) and 6.d), an event appears each 0.5 time unit, the event could be either a change of sign or the appearance of the unevenness marked by an arrow. This could explain why this frequency is important in the spectrograms. Finally, one can also observe that the fundamental frequency of the discussed behaviour is equal to $522 \mathrm{~Hz}(1306-784)$, which corresponds to the second partial of the signal. 


\subsubsection{Contact times}

In the centered obstacle configuration and according to the theoretical solution (see Section 3.2), persistent contact arises as the string is under its rest position. This section aims at confronting this result to experimental and numerical ones. To this end, the set up described in Section 4.1 .2 is employed. Fig. 17 shows experimental and numerical results. During the first periods, the contact is clear and persistent experimentally. However as time progresses, it becomes more confused, certainly because of dispersion which makes the waveform more complex.
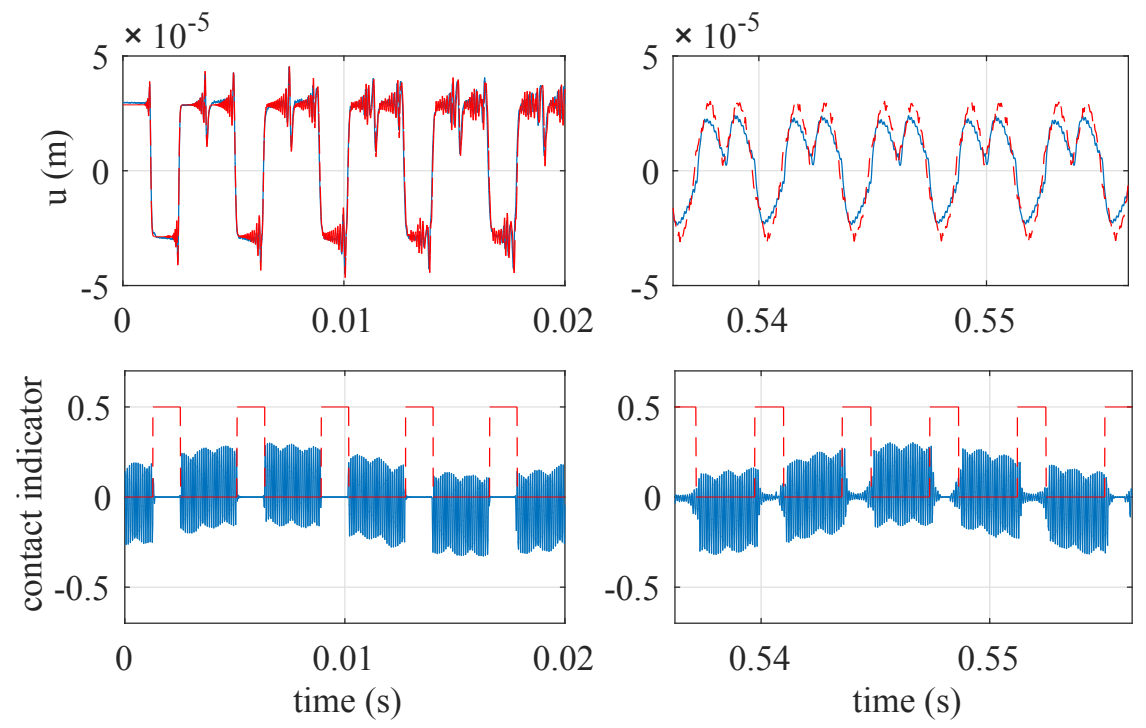

Figure 17: Top: experimental (blue line) and numerical (red dashed line) string displacement with a centered point obstacle, $B=1.78 \times 10^{-5}$. Bottom: experimental tension between the string and the obstacle (blue line) and numerical contact indicator function (red dashed line).

Numerical results have been obtained with the same string parameters as previously, $K=10^{13}$, $\alpha=1.5$ and $F_{s}=2 \mathrm{MHz}$. According to values of the contact indicator function, which equals 0.5 if contact arises and 0 otherwise, the contact is persistent. In fact, at the contact point, the string oscillates, however it does not make the contact indicator function switch since oscillations have an amplitude around $10^{-8} \mathrm{~m}$, which remains smaller than the penetration of the string in the obstacle, around $10^{-7} \mathrm{~m}$. The string thus oscillates under the rest position and contact detection remains positive. This behaviour is strongly related to the choice of $\alpha$ and $K$. Higher stiffness parameters affect the contact persistence by decreasing the allowed penetration. For instance with $\alpha=1.3$ and $K=10^{13}$, the penetration is about $10^{-8} \mathrm{~m}$ and first oscillations arise in the neighborhood of 0 , such that the indicator function oscillates at the beginning of each crenel.

\subsubsection{D string motion}

So far, the initial condition is given in the $(O z)$ direction only. An initial condition combining $(O z)$ and $(O y)$ polarisations is now considered in the case of a centered point obstacle. Numerical and experimental results are compared in Fig. 18, where numerical friction force parameters (see Section 2.7) are empirically determined as $s=10^{-5} \mathrm{~m} . \mathrm{s}^{-1}$ and $A=0.12 \mathrm{~N}$. Other parameters are unchanged.

The initial condition is similar to that used in the previous section except that approximately the same amplitude (about $1 \mathrm{~mm}$ ) is imposed along $(O z)$ and $(O y)$. The oscillation plane resulting from this initial condition is thus at 45 degrees in $(y O z)$.

Since the observation points are slightly displaced from one polarisation to the other, the displayed displacement $v$ has a larger amplitude. The main observation reported from Fig. 18 is the very fast decay of oscillations along $(O y)$, since the motion cancels out after $0.025 \mathrm{~s}$ while the motion along $(O z)$ continues during several seconds. The second comment is that the numerical scheme well reproduces details of the decay of the displacement along $(O y)$, excepted small disturbances (of a few $\mu \mathrm{m}$ ) when the string touches the obstacle, which also slightly affect the displacement along $(O z)$ and may be due to asperities on the obstacle which are not included in the model. 

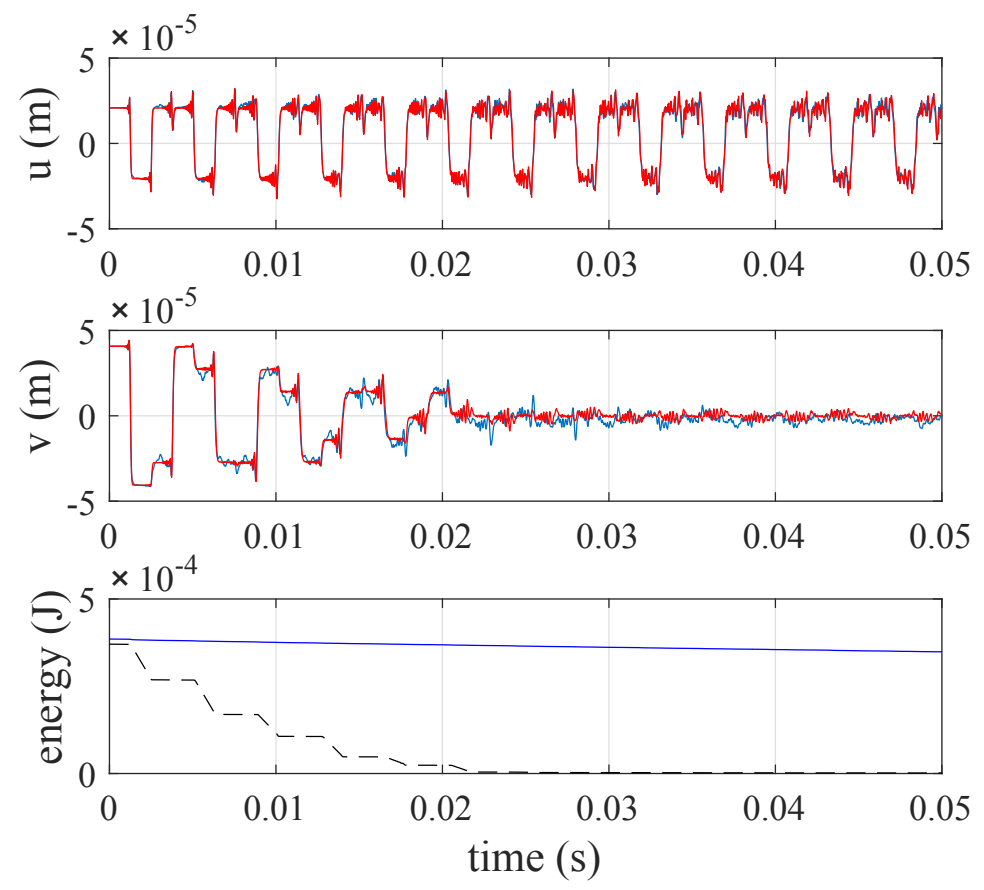

Figure 18: Top and centre: experimental (blue line) and numerical (red line) string displacement along $(O z)($ at $1 \mathrm{~cm}$ near the edge $x=L$ ) and $(O y)$ (at $2 \mathrm{~cm}$ near the edge $x=L$ ) with a centered point obstacle and $B=1.78 \times 10^{-5}$. Bottom: numerical energy of $u$ (blue line) and $v$ (dark dashed line).

As expected from displacement signals, the energy along $(O y)$ decreases rapidly. The largest amount of energy decrease arises when $u$ is negative, which corresponds to contact times, so that friction is applied on $v$.

\subsection{Two point bridge}

In this part, the bridge of a tanpura is modelled using a two point bridge constituted by a point obstacle near a boundary, as explained in [30, 13. The distance between the point obstacle and the string boundary $x=0$ is chosen as $x_{b}=6 \mathrm{~mm}$ according to the range of values given in [30] (5 to 7 $\mathrm{mm}$ for a string of length $1 \mathrm{~m}$ ).

Fig. 19 presents numerical and experimental signals in the case of a two point bridge. Again, the global shape of the signal as well as detailed oscillations are finely reproduced numerically. Effect of dispersion is faithfully described as can be seen on extended views at 0 and $0.2 \mathrm{~s}$ in particular. A slight amplitude error appears, smaller than in the centered obstacle case, as well as a slight delay (20 degrees after $1.5 \mathrm{~s}$ ). Possible reasons are the same as in the previous case (see Section 5.2). Besides, the total energy decreases faster than in the centered point obstacle case, itself decreasing faster than when there is no obstacle. This could be explained by an improved transfer of energy to the high-frequency range in the case of the two point bridge, where damping factors are larger.

Let us now focus on spectrograms (see Fig. 20). Note that only frequencies up to $4.8 \mathrm{kHz}$ are shown, contrarily to the case of the centered point obstacle. In the present case, no particular behaviour can be seen for higher frequencies, and the presented spectrograms focus on the zone of interest. As in the centered obstacle case, no missing mode is observed, due to the coupling of modes at the contact point. A descending formant can be observed which follows a time evolution as described in [30. (experimental study) and [34] (numerical study) where a string vibrating against a tanpura bridge is considered. Its evolution is accurately reproduced by the numerical result, although some differences appear after $2 \mathrm{~s}$, when the signal amplitude has become very small. The essential role of dispersion highlighted in [30, 14 is again demonstrated through the spectrogram in Fig. 21 where dispersion is cancelled. Comparing Fig. 20b and 21, one observes a similar ascending behaviour for lowest frequencies and during the first $1 \mathrm{~s}$ of the signal. However spectrograms substantially differ after in time as well as in the high frequency range. This shows the essential role of dispersion in the rich and complex behaviour of the signal. 


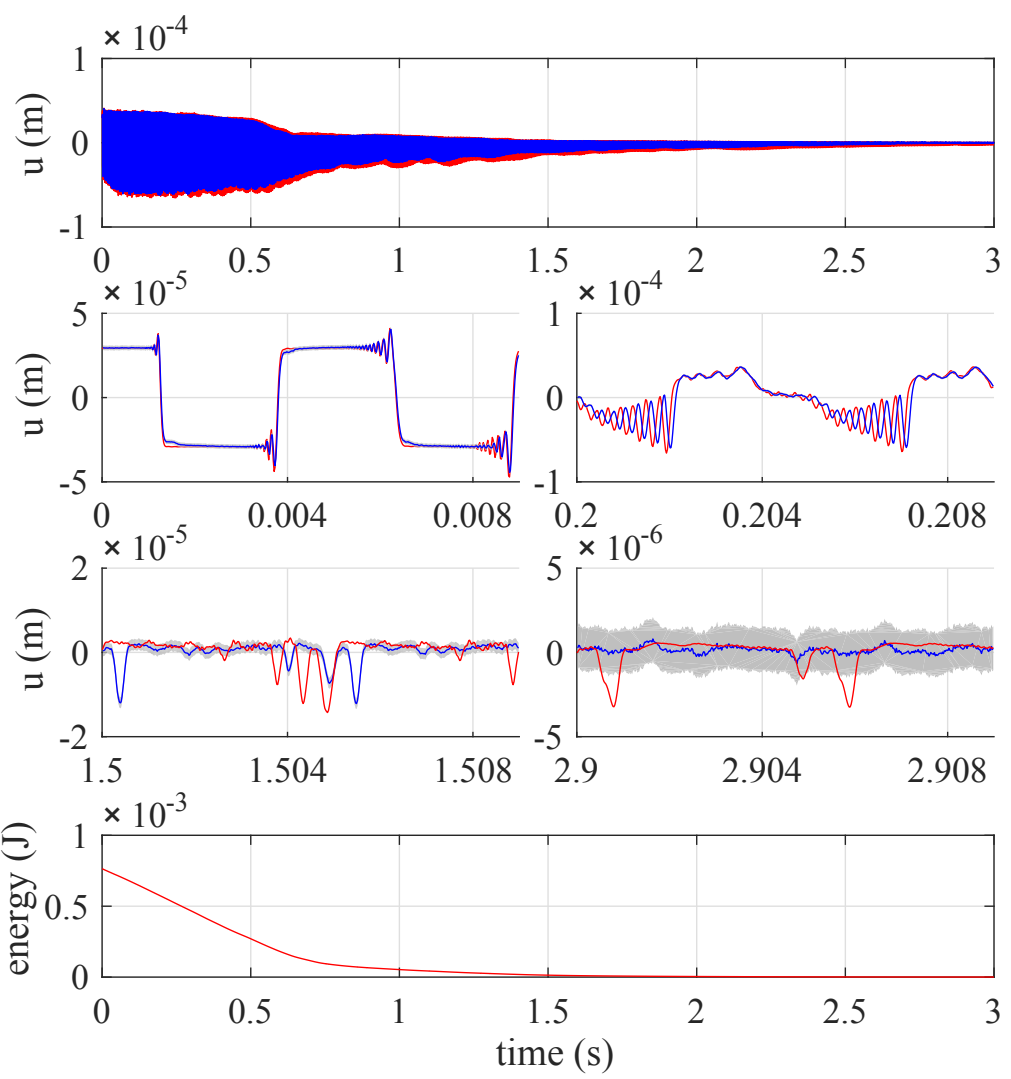

Figure 19: Displacement of the string when vibrating with a two point bridge, $B=1.78 \times 10^{-5}$. Comparison between measurement (blue line) and numerical simulation (red line), vertical displacement at $1 \mathrm{~cm}$ near the edge $x=L$. Expanded uncertainty at $95 \%$ (gray). Bottom shows the temporal decrease of the energy numerically computed.

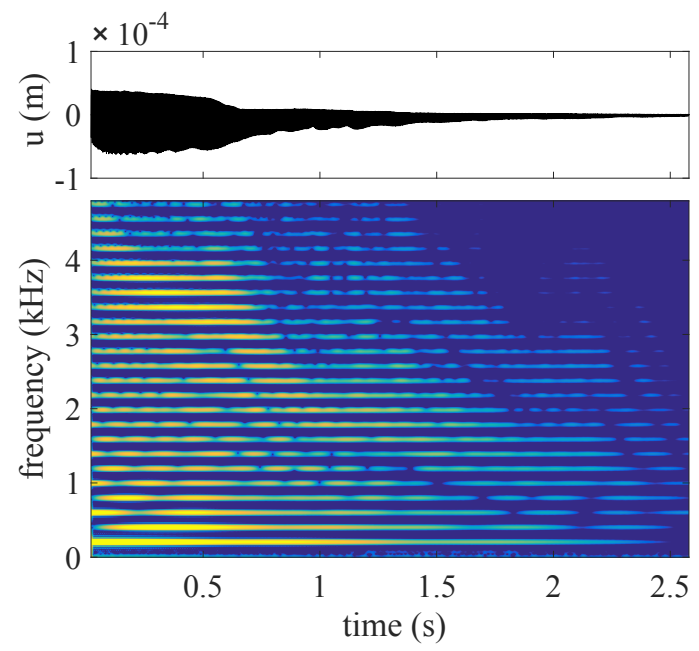

(a)

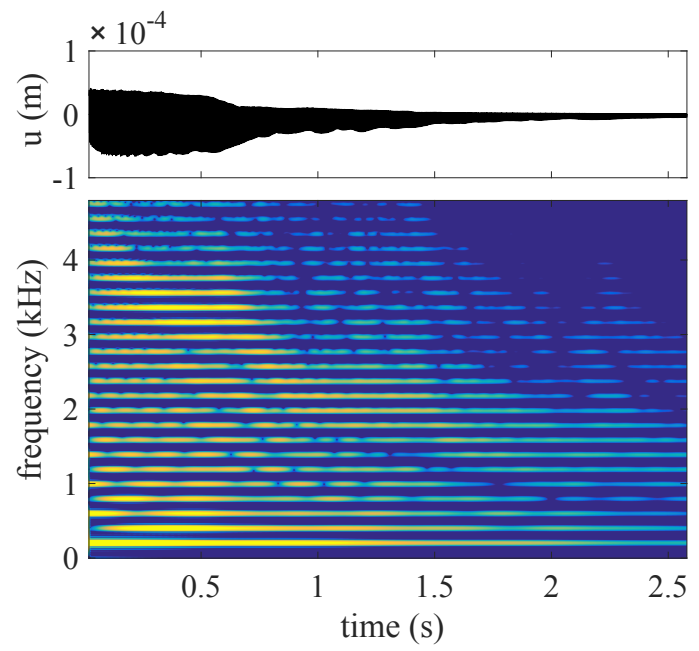

(b)

Figure 20: Two point bridge, $B=1.78 \times 10^{-5}$. Spectrograms: (a) experimental, (b) numerical.

\section{Conclusion}

In this paper, the motion of a stiff damped string against an obstacle has been studied numerically and experimentally in both transverse polarisations. The present investigations focus on point obstacles, but the scheme allows the consideration of arbitrarily shaped obstacles along $(O z)$. It is based on a modal approach, allowing a flexible adjustment of numerical behaviour in the linear regime (i.e., the eigenfrequencies and frequency-dependent damping coefficients). In particular, measured values 


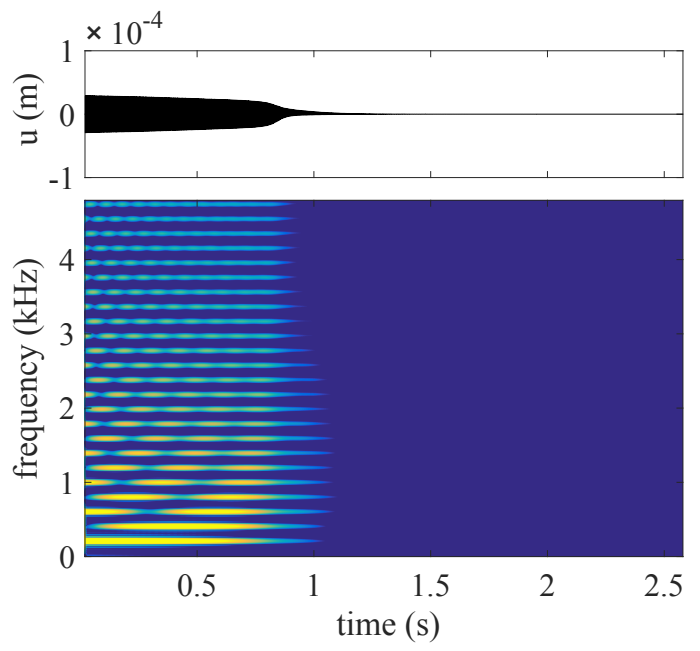

Figure 21: Two point bridge. Numerical spectrogram, without dispersion.

can be employed so that very realistic results can be obtained, which constitutes a major advantage of the method. While having a intrinsically modal nature, the scheme operates in the spatial domain. It could therefore be interpreted as a spectral method [57 combined with a time-stepping method. It is unconditionally stable, so that no bound on space and time steps is required for stability. Moreover, it is exact when the collision force is not present, contrary to other existing methods such as Hamiltonian methods [58, 33] and finite differences [46]. The necessity of a high sampling rate has been highlighted in order to obtain reliable results for simulations over a long duration. This affects the computation time, which could be improved by defining a variable spatial step, finer around the obstacle, and a variable time step, finer around contact events. Such refinements should however be carefully handled, since a variable space step would change the structure of involved matrices, and a variable time step should be completed together with a sampling rate conversion without introducing additional artefacts. The relevance of numerical results with regards to experiments has been demonstrated in Section 5 . To this end, a highly controlled experimental set up has been presented, as well as a reliable measure of the string linear features. Then a fine comparison between numerical and experimental results has been completed over a long duration, with an obstacle either at the middle of the string or near one boundary. In both cases, comparisons show an almost perfect agreement, without adding losses in the contact law. Results thus demonstrate both the accuracy of the numerical method and its ability to recover the most important physical features of the experiment. To the knowledge of authors, such a detailed comparison is absent from the literature.

In the present study, no realistic excitation mechanism in relation to musical gesture is included. The next step may thus be to incorporate the dynamics of the musician's fingers [59]. Moreover, differently shaped obstacles may be considered, including distributed barriers in order to simulate a wider range of musical instruments. In addition, the coupling between transverse motions of the string is limited and unilateral. A more complex model may be considered. In order to complete the model, coupling to the structure could also be included as well as possible sympathetic strings [60, 61.

\section{Acknowledgments}

This work was supported by the European Research Council, under grant number StG-2011-279068NESS. The authors also thank Laurent Quartier for contributing to the experimental set up realisation, as well as Benoit Fabre and Patrick Ballard for advices and discussions.

\section{AppendixA. Identities and inequalities for stability analysis}

In this appendix, useful properties of the matrices involved in the numerical scheme are demonstrated. Three main properties, directly used in the proof of the stability of the scheme, are shown. 
The following identities are also recalled for an $N \times N$ positive semi-definite symmetric matrix $\mathbf{M}$, and $\mathbf{u}^{n} \in \mathbb{R}^{N}$ a vector.

$$
\begin{aligned}
& \text { 1. }\left\langle\mathbf{M} \delta_{t t} \mathbf{u}^{n}, \delta_{t .} \mathbf{u}^{n}\right\rangle=\frac{1}{2} \delta_{t-}\left\langle\delta_{t+} \mathbf{u}^{n}, \mathbf{M} \delta_{t+} \mathbf{u}^{n}\right\rangle \\
& \text { 2. }\left\langle\mathbf{M} \mathbf{u}^{n}, \delta_{t .} \mathbf{u}^{n}\right\rangle=\frac{1}{2} \delta_{t-}\left\langle\mathbf{u}^{n+1}, \mathbf{M} \mathbf{u}^{n}\right\rangle
\end{aligned}
$$

These identities are useful in the course of the computations for demonstrating stability. The proof is straightforward.

Let us give the first property.

Property 1. Let $\mathbf{M}$ be a $N \times N$ positive semi-definite symmetric matrix and $\mathbf{u}^{n}, \mathbf{u}^{n+1} \in \mathbb{R}^{N}$. Then one has:

$$
\left\langle\mathbf{u}^{n+1}, \mathbf{M} \mathbf{u}^{n}\right\rangle \geq-\frac{\Delta t^{2}}{4}\left\langle\delta_{t+} \mathbf{u}^{n}, \mathbf{M} \delta_{t+} \mathbf{u}^{n}\right\rangle .
$$

Proof. Because $\mathbf{M}$ is symmetric, we have the following equality for any two vectors $\mathbf{u}, \mathbf{v}$ in $\mathbb{R}^{N}$ :

$$
\langle\mathbf{u}, \mathbf{M v}\rangle=\Delta x \sum_{i, k} m_{i k} v_{i} u_{k}=\Delta x \sum_{i, k} m_{i k} v_{k} u_{i}=\Delta x\langle\mathbf{v}, \mathbf{M u}\rangle .
$$

The inequality then results from the following equality:

$$
\left\langle\mathbf{u}^{n+1}, \mathbf{M} \mathbf{u}^{n}\right\rangle=\frac{1}{4}\left\langle\mathbf{u}^{n+1}+\mathbf{u}^{n}, \mathbf{M}\left(\mathbf{u}^{n+1}+\mathbf{u}^{n}\right)\right\rangle-\frac{\Delta t^{2}}{4}\left\langle\delta_{t+} \mathbf{u}^{n}, \mathbf{M} \delta_{t+} \mathbf{u}^{n}\right\rangle .
$$

Assuming that $\mathbf{M}$ is semi-definite positive gives the result.

Property 2. Using the notations defined in Section 2.6, $1+e_{i} \pm A_{i}>0, \forall i$.

Proof. Let us first consider the case $0<\sigma_{i}<\omega_{i}$.

Introducing $X=\sigma_{i} \Delta t, Y=\omega_{i} \Delta t$, and $Z=\sqrt{Y^{2}-X^{2}}$, one has:

$$
1+e_{i}-A_{i}=1+\mathrm{e}^{-2 X}-2 \mathrm{e}^{-X} \cos (Z)>1+\mathrm{e}^{-2 X}-2 \mathrm{e}^{-X}=f_{1}(X) .
$$

$f_{1}(X)$ is positive since $f_{1}^{\prime}(X)>0$ and $f_{1}(0)=0$, therefore $1+e_{i}-A_{i}>0$. The same reasoning leads to $1+e_{i}+A_{i}>0$.

Let us now study the case $0<\omega_{i}<\sigma_{i}$. In this case, defining $Z=\sqrt{X^{2}-Y^{2}}$ :

$$
1+e_{i}-A_{i}=1+\mathrm{e}^{-2 X}-2 \mathrm{e}^{-X} \cosh (Z)=f_{2}(X, Z) .
$$

By assumption, $0<\omega_{i}<\sigma_{i}$, so that $0<Y<X$, and therefore $0<Z<X$. On one hand, $f_{2, X}>2\left(\mathrm{e}^{-X}-\mathrm{e}^{-2 X}\right)>0$ and $f_{2}(0, Z)=0$ (since $0<Z<X$, the limiting case is $\left.X=Z=0\right)$. On the other hand, $f_{2, Z}=-2 \mathrm{e}^{-X} \sinh (Z)<0$ and $f_{2}(X, X)=0$. Finally, $1+e_{i}-A_{i}>0$. Obtaining $1+e_{i}+A_{i}>0$ is straightforward.

Property 3. $\check{\mathbf{D}}_{1}, \check{\mathbf{D}}_{2}$ and $\check{\mathbf{D}}_{3}$ (given in Section (2.6)) are symmetric and positive semi-definite.

Proof. The proof is given for $\check{\mathbf{D}}_{2}$ and $\check{\mathbf{D}}_{3}$. Given those, the proof for $\check{\mathbf{D}}_{\mathbf{1}}$ is straightforward. Let us first focus on $\check{\mathbf{D}}_{2}$.

Since $\check{\mathbf{C}}_{\mathbf{2}}$ is diagonal and $\tilde{\mathbf{S}}^{-1}=\tilde{\mathbf{S}}^{T}$, symmetry of $\check{\mathbf{D}}_{\mathbf{2}}$ is obtained by construction of the matrix:

$$
\check{\mathbf{D}}_{\mathbf{2}}=\mathbf{S} \check{\mathbf{C}}_{2} \mathbf{S}^{-1}=\Delta x \mathbf{S} \check{\mathbf{C}}_{2} \mathbf{S}^{T}
$$

of which coefficients are given by:

$$
\check{D}_{2_{i j}}=\Delta x \sum_{k=1}^{N-1} S_{i k} \check{C}_{2_{k k}} S_{k j}^{T}=\Delta x \sum_{k=1}^{N-1} S_{i k} \check{C}_{2_{k k}} S_{j k}
$$

with $i, j \in\{1, \ldots, N\}$. 
Let us now show that $\check{\mathbf{D}}_{\mathbf{2}}$ is positive semi-definite. To do so, it is sufficient to show that $\check{\mathbf{C}}_{\mathbf{2}}$ is positive semi-definite. Indeed:

Let $\mathbf{C}$ be a square diagonal matrix and $\mathbf{D}=\tilde{\mathbf{S}} \mathbf{C} \tilde{\mathbf{S}}^{-1}$ with $\tilde{\mathbf{S}}=\sqrt{\Delta x} \mathbf{S}$, where $\mathbf{S}$ is such that $\tilde{\mathbf{S}}^{-1}=\tilde{\mathbf{S}}^{T}$. Consider $\mathbf{q}$ and $\mathbf{u}$ such that $\mathbf{u}=\tilde{\mathbf{S}} \mathbf{q}$. Then: $\langle\mathbf{q}, \mathbf{C q}\rangle=\mathbf{q}^{T} \mathbf{C q}=\mathbf{q}^{T} \tilde{\mathbf{S}}^{T} \tilde{\mathbf{S}} \mathbf{C} \tilde{\mathbf{S}}^{T} \tilde{\mathbf{S}} \mathbf{q}=\langle\mathbf{u}, \mathbf{D u}\rangle$. Therefore, if $\mathbf{C}$ is positive semi-definite, $\mathbf{D}$ is.

If diagonal coefficients of $\check{\mathbf{C}}_{\mathbf{2}}$ are positive, then the proof is done. Since $\omega_{i}>0 \forall i$, one has to show that $1+\left(1-\gamma_{i}\right) \frac{\omega_{i}^{2} \Delta t^{2}}{2}+\sigma_{i}^{*} \Delta t>0$. Developing and rearranging this expression, one obtains:

$$
1+\left(1-\gamma_{i}\right) \frac{\omega_{i}^{2} \Delta t^{2}}{2}+\sigma_{i}^{*} \Delta t=\frac{\omega_{i}^{2} \Delta t^{2}}{2}\left(1+\frac{1-e_{i}}{1+e_{i}}\right)\left[\frac{1+e_{i}}{1+e_{i}-A_{i}}\right] .
$$

A.4 is positive if $1+e_{i}-A_{i}>0$. This is satisfied (see Property 2, so that $1+\left(1-\gamma_{i}\right) \frac{\omega_{i}^{2} \Delta t^{2}}{2}+\sigma_{i}^{*} \Delta t>0$.

Finally, $\check{\mathbf{D}}_{\mathbf{2}}$ is semi-definite positive. In the lossless case, which is a limiting case to the lossy one, the demonstration is similar, starting from a reduced expression of coefficients.

Let us now study $\check{\mathbf{D}}_{\mathbf{3}}$, the symmetry of which is obtained as previously.

As for $\check{\mathbf{D}}_{2}$, it is sufficient to show that $\check{\mathbf{C}}_{3}$ is semi-definite positive. The denominator of $\check{C}_{3_{i i}}$ is positive as demonstrated above. Besides, one has:

$$
\sigma_{i}^{*}=\frac{1-e_{i}}{1+e_{i}}\left[\frac{\omega_{i}^{2} \Delta t}{2}\left(1+\frac{A_{i}}{1+e_{i}-A_{i}}\right)\right]
$$

As previously, this quantity is positive. Finally, $\check{\mathbf{D}}_{\mathbf{3}}$ is semi-definite positive. In the lossless case, this matrix does not appear in the problem since it is null.

\section{References}

[1] B. Brogliato, Nonsmooth Mechanics, Springer Verlag, London, 1999.

[2] F. Pfeiffer, C. Glocker, Multibody Dynamics with Unilateral Contacts, Wiley VCH, 1996.

[3] H. Hertz, On the contact of elastic solids, Journal für die Reine und Andewandte Mathematik 29 (1881) $156-171$.

[4] D. Baraff, Fast contact force computation for nonpenetrating rigid bodies, in: SIGGRAPH, Orlando, 1994, pp. 23-24.

[5] F. Bertails-Descoubes, F. Cadoux, G. Davier, V. Acary, A nonsmooth Newton solver for capturing exact Coulomb friction in fiber assemblies, ACM Transactions on Graphics 30 (1) (2011) Article No. 6.

[6] T. L. H. Donahue, M. L. Hull, M. Rashid M., C. R. Jacobs, A finite element model of the human knee joint for the study of tibio-femoral contact, Journal of Biomechanical Engineering 124 (2002) $273-280$.

[7] F. Pfeiffer, C. Glocker, Contact in multibody systems, Journal of Applied Mathematics and Mechanics 64 (5) (2000) 773-782.

[8] A. Batailly, M. Legrand, A. Millecamps, F. Garcin, Numerical-experimental comparison in the simulation of rotor/stator interaction through blade-tip/abradable coating contact, Journal of Engineering for Gas Turbines and Power 134 (8) (2012) 082504.

[9] X. Boutillon, Model for piano hammers: Experimental determination and digital simulation, Journal of the Acoustical Society of America 83 (2) (1988) 746-754.

[10] A. Chaigne, P. Joly, L. Rhaouti, Numerical modeling of the timpani, in: European Congress on Computational Methods in Applied Sciences and Engineering, Barcelona, 2000.

[11] S. Bilbao, A. Torin, V. Chatziioannou, Numerical modeling of collisions in musical instruments, Acta Acustica united with Acustica 101 (2015) 155-173. 
[12] C. Raman, On some indian stringed instruments, Indian Association for the Cultivation of Science (7) (1921) 29-33.

[13] C. Valette, C. Cuesta, C. Besnainou, M. Castellengo, The tampura bridge as a precursive wave generator, Acustica 74 (1991) 201-208.

[14] S. Siddiq, A physical model of the nonlinear sitar string, Archives of acoustics 37 (1) (2012) 73-79.

[15] L. Trautmann, R. Rabenstein, Multirate simulations of string vibrations including nonlinear fretstring interactions using the functional transformation method, EURASIP Journal on Applied Signal Processing 7 (2004) 949-963.

[16] S. Bilbao, A. Torin, Numerical simulation of string/barrier collisions: the fretboard, in: International Conference on Digital Audio Effects, DAFx-14, Erlangen, 2014.

[17] L. Amerio, Continuous solutions of the problem of a string vibrating against an obstacle, Rendiconti del Seminario Matematico della Università di Padova 59 (1978) 67-96.

[18] H. Cabannes, Cordes vibrantes avec obstacles (Vibrating strings with obstacles), Acustica 55 (1984) $14-20$.

[19] H. Cabannes, Presentation of software for movies of vibrating strings with obstacles, Applied Mathematics Letters 10 (5) (1996) 79-84.

[20] M. Schatzman, A hyperbolic problem of second order with unilateral constraints: the vibrating string with a concave obstacle, Journal of Mathematical Analysis and Applications 73 (1980) $138-191$.

[21] R. Burridge, J. Kappraff, C. Morshedi, The sitar string, a vibrating string with a one-sided inelastic constraint, SIAM Journal of Applied Mathematics 42 (6) (1982) 1231-1251.

[22] M. Schatzman, Un problème hyperbolique du 2ème ordre avec contrainte unilatérale : La corde vibrante avec obstacle ponctuel (A hyperbolic problem of second order with unilateral constraints: the vibrating string with a point obstacle), Journal of Differential Equations 36 (1980) 295-334.

[23] E. Rank, G. Kubin, A waveguide model for slapbass synthesis, in: IEEE International Conference on Acoustics, Speech, and Signal Processing, Munich, Vol. 1, 1997, pp. 443-446.

[24] G. Evangelista, F. Eckerholm, Player-instrument interaction models for digital waveguide synthesis of guitar: Touch and collisions, IEEE transactions on audio, speech, and language processing 18 (4) (2010) 822-832.

[25] D. Kartofelev, A. Stulov, H.-M. Lehtonen, V. Välimäki, Modeling a vibrating string terminated against a bridge with arbitrary geometry, in: Stockholm Music Acoustics Conference, 2013.

[26] A. Krishnaswamy, J. O. Smith, Methods for simulating string collisions with rigid spatial objects, in: IEEE Workshop of Applications of Signal Processing to Audio and Acoustics, New Paltz, NY, 2003, pp. 233-236.

[27] C. P. Vyasarayani, S. Birkett, J. McPhee, Modeling the dynamics of a vibrating string with a finite distributed unilateral constraint: Application to the sitar, Journal of the Acoustical Society of America 125 (6) (2009) 3673-3682.

[28] A. K. Mandal, P. Wahi, Natural frequencies, modeshapes and modal interactions fot strings vibrating against an obstacle: Relevance to sitar and veena, Journal of Sound and Vibration 338 (2015) 42-59.

[29] C. P. Vyasarayani, Transient dynamics of continuous systems with impact and friction, with applications to musical instruments, Ph.D. thesis, University of Waterloo (2009).

[30] C. Valette, C. Cuesta, Mécanique de la corde vibrante (Mechanics of the vibrating string), Hermès, Paris, 1993. 
[31] D. J. Wagg, A note on coefficient of restitution models including the effects of impact induced vibration, Journal of Sound and Vibration 300 (2007) 1071-1078.

[32] C. P. Vyasarayani, J. McPhee, S. Birkett, Modeling impacts between a continuous system and a rigid obstacle using coefficient of restitution, ASME Journal of Applied Mechanics 77 (2010) 021008:1-7.

[33] V. Chatziioannou, M. Van Walstijn, Energy conserving schemes for the simulation of musical instrument contact dynamics, Journal of Sound and Vibration 339 (2015) 262-279.

[34] M. Van Walstijn, V. Chatziioannou, Numerical simulation of tanpura string vibrations, in: International Symposium on Musical Acoustics, ISMA 2014, Le Mans, 2014.

[35] C. Desvages, S. Bilbao, Two-polarisation finite difference model of bowed strings with nonlinear contact and friction forces, in: International Conference on Digital Audio Effects, DAFx-15, Trondheim, 2015.

[36] J. Bridges, M. Van Walstijn, Investigation of tanpura string vibrations using a two-dimensional time-domain model incorporating coupling and bridge friction, in: Vienna Talk on Music Acoustics, Vienna, 2015.

[37] V. Astashev, V. Krupenin, Experimental investigation of vibrations of strings interaction with point obstacles, Doklady Physics 46 (7) (2001) 522-525.

[38] T. Taguti, Y. Tohnai, Acoustical analysis on the sawari tone of chikuzen biwa, Acoustical Science and Technology 22 (3) (2001) 199-207.

[39] S. Weisser, O. Lartillot, Investigating non-western musical timbre: a need for joint approaches, in: Third International Workshop on Folk Music Analysis, Amsterdam, 2013, pp. 33-39.

[40] C. Cuesta, C. Valette, Théorie de la corde pincée en approximation linéaire (Theory of the plucked string using the linear approximation), Acustica 71 (1990) 28-40.

[41] B. Brogliato, V. Acary, Numerical Methods for Nonsmooth Dynamical Systems. Applications in Mechanics and Electronics. Lecture Notes in Applied and Computational Mechanics, Vol. 35 of Lecture Notes in Applied and Computational Mechanics, Springer Verlag, Berlin Heidelberg, 2008.

[42] C. Studer, Numerics of unilateral contacts and friction, Vol. 47 of Lecture Notes in Applied and Computational Mechanics, Springer Verlag, Berlin Heidelberg, 2009.

[43] W. Goldsmith, Impact, Dover publications, Mineola, 2001.

[44] M. Machado, P. Moreira, P. Flores, H. M. Lankarani, Compliant contact force models in multibody dynamics: Evolution of the Hertz contact theory, Mechanism and Machine Theory 53 (2012) 99121.

[45] A. Banerjee, A. Chanda, R. Das, Historical origin and recent development on normal directional impact models for rigid body contact simulation: A critical review, Archives of Computational Methods in Engineering (2016) 1-26.

[46] S. Bilbao, Numerical Sound Synthesis: Finite Difference Schemes and Simulation in Musical Acoustics, Wiley, Chichester, 2009.

[47] A. Chaigne, A. Askenfelt, Numerical simulations of piano strings. I. a physical model for a struck string using finite difference methods, Journal of the Acoustical Society of America 95 (2) (1994) $1112-1118$.

[48] M. Ducceschi, C. Touzé, Modal approach for nonlinear vibrations of damped impacted plates: Application to sound synthesis of gongs and cymbals, Journal of Sound and Vibration 344 (2015) 313-331.

[49] A. Paté, J.-L. Le Carrou, B. Fabre, Predicting the decay time of solid body electric guitar tones, Journal of the Acoustical Society of America 135 (5) (2014) 3045-3055. 
[50] K. Hunt, F. Crossley, Coefficient of restitution interpreted as damping in vibroimpact, Journal of Applied Mechanics (1975) 440-445.

[51] S. Bilbao, Numerical modeling of string/barrier collisions, in: International Symposium on Musical Acoustics, ISMA 2014, Le Mans, 2014.

[52] H. Cabannes, Mouvements presque-périodiques d'une corde vibrante en présence d'un obstacle fixe, rectiligne ou ponctuel (Almost periodic motion of a string vibrating against a straight or point fixed obstacle), International Journal of Non-linear Mechanics 16 (5/6) (1981) 449-458.

[53] C. Cuesta, C. Valette, Evolution temporelle de la vibration des cordes de clavecin (Temporal evolution of harpsichord strings vibration), Acustica 66 (1988) 37-45.

[54] J.-L. Le Carrou, D. Chadefaux, L. Seydoux, B. Fabre, A low-cost high-precision measurement method of string motion, Journal of Sound and Vibration 333 (2014) 3881-3888.

[55] R. Roy, A. Paulraj, T. Kailath, Esprit - a subspace rotation approach to estimation of parameters of cisoids in noise, IEEE transactions on acoustics, speech, and signal processing 34 (5) (1986) $1340-1342$.

[56] J.-L. Le Carrou, F. Gautier, R. Badeau, Sympathetic string modes in the concert harp, Acta Acustica united with Acustica 95 (4) (2009) 744-752.

[57] L. N. Trefethen, Spectral Methods in MATLAB, SIAM, Philadelphia, PA, 2000.

[58] T. Falaize, T. Hélie, Guaranteed-passive simulation of an electro-mechanical piano: a portHamiltonian approach, in: International Conference on Digital Audio Effects, DAFx-15, Trondheim, 2015.

[59] D. Chadefaux, J.-L. Le Carrou, B. Fabre, A model of harp plucking, Journal of the Acoustical Society of America 133 (4) (2013) 2444-2455.

[60] S. Weisser, M. Demoucron, Shaping the resonance. sympathetic strings in hindustani classical instruments, in: Meetings on Acoustics, Vol. 15, 2012.

[61] J.-L. Le Carrou, F. Gautier, N. Dauchez, J. Gilbert, Modelling of sympathetic string vibrations, Acta Acustica united with Acustica 91 (2) (2005) 277-288. 